\title{
Barnes-Ismagilov Integrals and Hypergeometric Functions of the Complex Field
}

Yury A. NERETIN $\dagger^{1} \dagger^{2} \dagger^{3} \dagger^{4}$

$\dagger^{1}$ Wolfgang Pauli Institut, c/o Fakultät für Mathematik, Universität Wien, Oskar-Morgenstern-Platz 1, A-1090 Wien, Austria

E-mail: yurii.neretin@univie.ac.at

URL: http://mat.univie.ac.at/ neretin/

$\dagger^{2}$ Institute for Theoretical and Experimental Physics, Moscow, Russia

$\dagger^{3}$ Faculty of Mechanics and Mathematics, Lomonosov Moscow State University, Russia

$\dagger^{4}$ Institute for Information Transmission Problems, Moscow, Russia

Received April 09, 2020, in final form July 17, 2020; Published online August 02, 2020

https://doi.org/10.3842/SIGMA.2020.072

Abstract. We examine a family ${ }_{p} G_{q}^{\mathbb{C}}\left[\begin{array}{c}(a) \\ (b)\end{array} ; z\right]$ of integrals of Mellin-Barnes type over the space $\mathbb{Z} \times \mathbb{R}$, such functions $G$ naturally arise in representation theory of the Lorentz group. We express ${ }_{p} G_{q}^{\mathbb{C}}(z)$ as quadratic expressions in the generalized hypergeometric functions ${ }_{p} F_{q-1}$ and discuss further properties of the functions ${ }_{p} G_{q}^{\mathbb{C}}(z)$.

Key words: Mellin-Barnes integrals; Mellin transform; hypergeometric functions; Lorentz group

2020 Mathematics Subject Classification: 33C20; 33C70; 22E43

\section{The statements}

\subsection{Introduction}

Recall the Euler integral representation of the Gauss hypergeometric function:

$$
{ }_{2} F_{1}\left[\begin{array}{c}
a, b \\
c
\end{array} ; z\right]=\frac{1}{\mathrm{~B}(b, c-b)} \int_{0}^{1} t^{b-1}(1-t)^{c-b-1}(1-z t)^{-a} \mathrm{~d} t,
$$

where $a, b, c$ are complex numbers and

$$
\mathrm{B}(\alpha, \beta):=\int_{0}^{1} t^{\alpha-1}(1-t)^{\beta-1} \mathrm{~d} t=\frac{\Gamma(\alpha) \Gamma(\beta)}{\Gamma(\alpha+\beta)},
$$

is the beta function. The hypergeometric functions ${ }_{p+1} F_{p}$ admit the following inductive integral representation:

$$
{ }_{p+1} F_{p}\left[\begin{array}{c}
a_{1}, \ldots, a_{p}, c \\
b_{1}, \ldots, b_{p-1}, d
\end{array} ; z\right]:=\frac{1}{\mathrm{~B}(c, d-c)} \int_{0}^{1} t^{c-1}(1-t)^{d-c-1}{ }_{p} F_{p-1}\left[\begin{array}{c}
a_{1}, \ldots, a_{p} \\
b_{1}, \ldots, b_{p-1}
\end{array} ; t z\right] \mathrm{d} t .
$$

Let us replace the integration over the interval $[0,1]$ by the integration over the complex plane for all integrals (1.1)-(1.3). The expression $t^{b}$ for $t \in \mathbb{C}, b \in \mathbb{C}$ is ramified at $t=0$, so the

This paper is a contribution to the Special Issue on Elliptic Integrable Systems, Special Functions and Quantum Field Theory. The full collection is available at https://www.emis.de/journals/SIGMA/elliptic-integrablesystems.html 
integration makes no sense. But we can replace $t^{b}$ by $|t|^{2 b}=t^{b} \bar{t}^{b}$. It is better to consider a wider generality and to write

$$
t^{b \mid b^{\prime}}:=t^{b} \bar{t}^{b^{\prime}} .
$$

This expression is non-ramified if $b-b^{\prime} \in \mathbb{Z}$. Then the new beta-function equals (see [17, Section II.3.7], [16, Section 1.6]) $)^{1}$

$$
\begin{aligned}
\mathrm{B}^{\mathbb{C}}\left(\alpha\left|\alpha^{\prime}, \beta\right| \beta^{\prime}\right): & =\frac{1}{\pi} \int_{\mathbb{C}} t^{\alpha-1 \mid \alpha^{\prime}-1}(1-t)^{\beta-1 \mid \beta^{\prime}-1} \mathrm{~d} \operatorname{Re} t \mathrm{~d} \operatorname{Im} t \\
& =\frac{\Gamma(\alpha) \Gamma(\beta)}{\Gamma(\alpha+\beta)} \cdot \frac{\Gamma\left(1-\alpha^{\prime}-\beta^{\prime}\right)}{\Gamma\left(1-\alpha^{\prime}\right) \Gamma\left(1-\beta^{\prime}\right)} .
\end{aligned}
$$

The new version of the Gauss hypergeometric function (Gauss hypergeometric functions of the complex field) also can be evaluated (see [33, Theorem 3.9]), the result has the form

$$
\begin{aligned}
& { }_{2} F_{1}^{\mathbb{C}}\left[\begin{array}{c}
a\left|a^{\prime}, b\right| b^{\prime} \\
c \mid c^{\prime}
\end{array} ; z={ }_{2} F_{1}\left[\begin{array}{c}
a, b \\
c
\end{array} ;\right]{ }_{2} F_{1}\left[\begin{array}{c}
a^{\prime}, b^{\prime} \\
c^{\prime}
\end{array} \bar{z}\right]\right. \\
& +\left\{\begin{array}{c}
\text { product of } \\
\text { T-functions }
\end{array}\right\} z^{1-c \mid 1-c^{\prime}}{ }_{2} F_{1}\left[\begin{array}{c}
a+1-c, b+1-c \\
2-c
\end{array} z\right]{ }_{2} F_{1}\left[\begin{array}{c}
a^{\prime}+1-c^{\prime}, b^{\prime}+1-c^{\prime} ; \bar{z} \\
2-c^{\prime}
\end{array}\right.
\end{aligned}
$$

The Gauss hypergeometric functions satisfy numerous identities, see for instance the book Higher Hypergeometric Functions [13, Chapter 2]. Usually such identities (and maybe all such identities) have counterparts for ${ }_{2} F_{1}^{\mathbb{C}}$ (see a collection of formulas in [33, Section 3]).

Counterparts of the Gauss hypergeometric functions were briefly mentioned in the book by Gelfand, Graev and Vilenkin [17, Section II.3.7] in 1962. Later various formulas and identities related to hypergeometric functions of complex fields of different levels and beta integrals of the complex field appeared in works of numerous authors: Dotsenko and Fateev [12], Gelfand, Graev and Retakh [16], Ismagilov [23, 24], Derkachov and Manashov [6, 8], Bazhanov, Mangazeev and Sergeev [2], Derkachov, Manashov, Valinevich [9, 10], Kels [27, 28], Mimachi [32], Derkachov and Spiridonov [11], Molchanov and Neretin [33], Neretin [39] (we discuss the references in a more arranged form in Section 3.3).

The purpose of this paper is to give a definition of counterparts of higher hypergeometric functions ${ }_{p} F_{q}(z)$ (formula (1.15)) and to obtain an analog of formula (1.5), see Theorem 1.2. The main statements are contained in the present section, their proofs are done in Section 2. In Section 3 we discuss further properties of our functions.

\subsection{Notation}

Denote by $\mathbb{Z}_{+}$the set of integers $\geqslant 0$, by $\mathbb{Z}_{-}$the set of integers $\leqslant 0$.

\subsubsection{Hypergeometric functions.}

Let $r \leqslant s+1$. Let $a_{1}, \ldots, a_{r} \in \mathbb{C}$ and $b_{1}, \ldots, b_{s} \in \mathbb{C} \backslash \mathbb{Z}_{-}$. Generalized hypergeometric functions are defined by

$$
{ }_{r} F_{s}\left[\begin{array}{l}
a_{1}, \ldots, a_{r} \\
b_{1}, \ldots, b_{r}
\end{array} ; z\right]:=\sum_{m=0}^{\infty} \frac{\left(a_{1}\right)_{m} \cdots\left(a_{r}\right)_{m}}{m !\left(b_{1}\right)_{m} \cdots\left(b_{s}\right)_{m}} \cdot z^{n},
$$

where $(a)_{m}:=a(a+1) \cdots(a+m-1)$ is the Pochhammer symbol. For $r \leqslant s$ the radius of convergence is $\infty$, for $r=s+1$ it is 1 . For $r>s+1$ the series diverges.

\footnotetext{
${ }^{1}$ To evaluate this integral, we can pass to polar coordinates $t=r \mathrm{e}^{\mathrm{i} \varphi}$. Integrating in $\varphi$ we get a piece-wise ${ }_{2} F_{1}$-expression in $r$ with a singularity at $r=1$. It remains to apply the Slater theorem, see [43, Theorem 4.8.1, namely formula (4.8.7)]. On the other hand, we can reduce (1.4) to the gamma-function (1.7) in the usual way (see Jacobi's proof of the beta-integral (1.2) in [1, Section I.1]), but a justification of a changing of integration order is unexpectedly tedious.
} 


\subsubsection{Notation for lists}

Let $a_{1}, \ldots, a_{p} \in \mathbb{C}$ and $h \in \mathbb{C}$. We use the following notation for lists,

$$
\begin{aligned}
& (a):=a_{1}, \ldots, a_{p} ; \\
& (a)+h:=a_{1}+h, \ldots, a_{p}+h ; \\
& (a)_{\backslash j}:=a_{1}, \ldots, a_{j-1}, a_{j+1}, \ldots, a_{p} .
\end{aligned}
$$

In particular, we denote $(1.6)$ by ${ }_{r} F_{s}\left[\begin{array}{l}(a) \\ (b)\end{array} ; z\right]$.

\subsubsection{Double powers}

Denote by $\mathbb{C}^{\times}$(resp. $\mathbb{R}_{+}^{\times}$) the multiplicative group of $\mathbb{C}$ (resp. the multiplicative group of positive reals). By $\mathbb{T} \subset \mathbb{C}^{\times}$we denote the subgroup $|z|=1$, we have $\mathbb{T} \simeq \mathbb{R} / 2 \pi \mathbb{Z}$,

$$
\mathbb{C}^{\times}=\mathbb{T} \times \mathbb{R}_{+}^{\times},
$$

and $\mathbb{R}_{+}^{\times}$is isomorphic to the additive group $\mathbb{R}$ of real numbers.

The dual group $\left(\mathbb{C}^{\times}\right)^{*}$, i.e., the group of all homomorphisms $\mathbb{C}^{\times} \rightarrow \mathbb{T}$, is

$$
\left(\mathbb{C}^{\times}\right)^{*}=(\mathbb{T})^{*} \times\left(\mathbb{R}_{+}^{\times}\right)^{*} \simeq \mathbb{Z} \times \mathbb{R} .
$$

Denote by $\Lambda_{\mathbb{C}}$ the set of pairs $a \mid a^{\prime}$ such that $a, a^{\prime} \in \mathbb{C}$ and $a-a^{\prime} \in \mathbb{Z}$. We write such pairs by

$$
a\left|a^{\prime}=\frac{k+\sigma}{2}\right| \frac{-k+\sigma}{2}, \quad \text { where } k \in \mathbb{Z}, \sigma \in \mathbb{C} .
$$

By $\Lambda \subset \Lambda_{\mathbb{C}}$ we denote the subset consisting of $a \mid a^{\prime}$ satisfying $a^{\prime}+\bar{a}=0$,

$$
a\left|a^{\prime}=\frac{k+\mathrm{i} s}{2}\right| \frac{-k+\mathrm{i} s}{2} \in \Lambda, \quad \text { where } s \in \mathbb{R} .
$$

Define the following functions on $\mathbb{C}^{\times}$:

$$
z^{a \mid a^{\prime}}:=z^{a} \bar{z}^{a^{\prime}}:=(z / \bar{z})^{k / 2}|z|^{\sigma} .
$$

Notice that such functions are precisely all homomorphisms $\mathbb{C}^{\times} \rightarrow \mathbb{C}^{\times}$. For $a \mid a^{\prime} \in \Lambda$ we get homomorphisms $\mathbb{C}^{\times} \rightarrow \mathbb{T}$,

$$
\left|z^{\frac{k+\mathrm{i} s}{2}}\right| \frac{-k+\mathrm{i} s}{2} \mid=1
$$

\subsubsection{The complex Mellin transform}

Denote by $\mathrm{d} \overline{\bar{t}}$ the Lebesgue measure on $\mathbb{C}$,

$$
\mathrm{d} \overline{\bar{t}}:=\mathrm{d} \operatorname{Re} t \mathrm{~d} \operatorname{Im} t .
$$

We define the complex Mellin transform $\mathcal{M}$ as the Fourier transform on the group $\mathbb{C}^{\times}$. For a function $f$ on $\mathbb{C}^{\times}$we define the Mellin transform as a function on $\Lambda_{\mathbb{C}}$ defined by

$$
g\left(a \mid a^{\prime}\right)=\mathcal{M} f\left(a \mid a^{\prime}\right):=\frac{1}{2 \pi} \int_{\mathbb{C}^{\times}} t^{a \mid a^{\prime}} f(t) \frac{\mathrm{d} \overline{\bar{t}}}{t^{1 \mid 1}},
$$

the factor $\mathrm{d} \overline{\bar{t}} / t^{1 \mid 1}$ is the $\mathbb{C}^{\times}$-invariant measure on $\mathbb{C}^{\times}$. 
The Mellin transform is a unitary operator from $L^{2}\left(\mathbb{C}^{\times}, \mathrm{d} \overline{\bar{t}} / t^{1 \mid 1}\right)$ to $L^{2}$ on $\Lambda \simeq \mathbb{Z} \times \mathbb{R}$, the inversion formula is

$$
f(t)=\frac{1}{2 \pi} \sum_{k \in \mathbb{Z}} \int_{\mathbb{R}} g\left(\frac{k+\mathrm{i} s}{2} \mid \frac{-k+\mathrm{i} s}{2}\right) t^{\frac{-k-\mathrm{i} s}{2}} \mid \frac{k-\mathrm{i} s}{2} \mathrm{~d} s .
$$

The convolution on the group $\mathbb{C}^{\times}$is defined by formula

$$
f * g(z):=\int_{\mathbb{C}} f(t) g(z / t) \frac{\mathrm{d} \overline{\bar{t}}}{t^{1 \mid 1}}
$$

The Mellin transform sends convolutions to products,

$$
\mathcal{M}(f * g)(z)=2 \pi \mathcal{M} f(z) \cdot \mathcal{M} g(z) .
$$

\subsection{The Gamma-function of the complex field}

Following Gelfand, Graev and Retakh [16], we define the Gamma-function of the complex field as a function on $\Lambda_{\mathbb{C}}$ by $^{2}$

$$
\begin{aligned}
\Gamma^{\mathbb{C}}\left(a \mid a^{\prime}\right) & :=\frac{1}{\pi} \int_{\mathbb{C}} t^{a-1 \mid a^{\prime}-1} \mathrm{e}^{2 \mathrm{i} \operatorname{Im} t} \mathrm{~d} \overline{\bar{t}} a \\
& =\frac{1}{\pi} \lim _{r \rightarrow \infty} \int_{|t| \leqslant r} t^{a-1 \mid a^{\prime}-1} \mathrm{e}^{2 \mathrm{i} \operatorname{Im} t} \mathrm{~d} \overline{\bar{t}}=\frac{\Gamma(a)}{\Gamma\left(1-a^{\prime}\right)}=\frac{(-1)^{a-a^{\prime}} \Gamma\left(a^{\prime}\right)}{\Gamma(1-a)} \\
& =\frac{1}{\pi} \Gamma(a) \Gamma\left(a^{\prime}\right) \sin \pi a^{\prime}=\frac{(-1)^{a-a^{\prime}}}{\pi} \Gamma(a) \Gamma\left(a^{\prime}\right) \sin \pi a .
\end{aligned}
$$

The integral conditionally converges if $0<\operatorname{Re}\left(a+a^{\prime}\right)<1$ and diverges otherwise. Clearly, the right hand side is meromorphic in the whole $\Lambda_{\mathbb{C}}$.

Remark 1.1. In particular, we can write (1.4) as

$$
\mathrm{B}^{\mathbb{C}}\left(\alpha\left|\alpha^{\prime}, \beta\right| \beta^{\prime}\right)=\frac{\Gamma^{\mathbb{C}}\left(\alpha \mid \alpha^{\prime}\right) \Gamma^{\mathbb{C}}\left(\beta \mid \beta^{\prime}\right)}{\Gamma^{\mathbb{C}}\left(\alpha+\beta \mid \alpha^{\prime}+\beta^{\prime}\right)}
$$

It is easy to see that

$$
\begin{aligned}
& \Gamma^{\mathbb{C}}\left(a \mid a^{\prime}\right)=(-1)^{a-a} \Gamma^{\mathbb{C}}\left(a^{\prime} \mid a\right), \\
& \Gamma^{\mathbb{C}}\left(a \mid a^{\prime}\right) \Gamma^{\mathbb{C}}\left(1-a \mid 1-a^{\prime}\right)=(-1)^{a-a^{\prime}}, \\
& \Gamma^{\mathbb{C}}\left(a+m \mid a^{\prime}+m^{\prime}\right)=(-1)^{m^{\prime}} \Gamma^{\mathbb{C}}\left(a \mid a^{\prime}\right)(a)_{m}\left(a^{\prime}\right)_{m^{\prime}}, \\
& \Gamma^{\mathbb{C}}\left(a-m \mid a^{\prime}-m^{\prime}\right)=\frac{(-1)^{m} \Gamma^{\mathbb{C}}\left(a \mid a^{\prime}\right)}{(1-a)_{m}\left(1-a^{\prime}\right)_{m^{\prime}}}, \\
& \prod_{j=0}^{k-1} \Gamma^{\mathbb{C}}\left(a+\frac{j}{k} \mid a^{\prime}+\frac{j}{k}\right)=\Gamma^{\mathbb{C}}\left(k a \mid k a^{\prime}\right) k^{1-\left(a+a^{\prime}\right) k},
\end{aligned}
$$

where $m \in \mathbb{N}, k \in \mathbb{N}$. Values of $\Gamma^{\mathbb{C}}$ at integer points are

$$
\Gamma^{\mathbb{C}}(k \mid l)=0, \quad \text { for } k, l \in \mathbb{N} ;
$$

\footnotetext{
${ }^{2}$ The definition slightly differs from a definition from [16], which was used in [33, 39]. We write $\operatorname{Im} t$ instead of Re $t$. For this reason the factor $i^{a-a^{\prime}}$ from [16, 33] disappears.
} 


$$
\Gamma(m \mid-k)=\frac{(m-1) !(-1)^{k}}{k !}, \quad \text { for } m \in \mathbb{N}, k \in \mathbb{Z}_{+} .
$$

For $m, m^{\prime} \in \mathbb{Z}_{+}$we have a pole at $(-m) \mid\left(-m^{\prime}\right)$, more precisely,

$$
\underset{\varepsilon=0}{\operatorname{res}} \Gamma^{\mathbb{C}}\left(-m+\varepsilon \mid-m^{\prime}+\varepsilon\right)=\frac{(-1)^{m}}{m ! m^{\prime} !} .
$$

Next (see Section 2.1 below), for $a \mid a^{\prime} \in \Lambda_{\mathbb{C}}$ and $\xi=\frac{1}{2}(k+\mathrm{i} s)$ with $k \in \mathbb{Z}, s \in \mathbb{R}$ we have

$$
\Gamma^{\mathbb{C}}\left(a+\xi \mid a^{\prime}-\bar{\xi}\right)=\exp \{2 \mathrm{i} \operatorname{Im}(\xi \ln \xi-\xi)\} \cdot \xi^{a-\frac{1}{2}} \mid a^{\prime}-\frac{1}{2}\left(1+O\left(|\xi|^{-1}\right)\right) .
$$

Therefore,

$$
\left|\Gamma^{\mathbb{C}}\left(a+\xi \mid a^{\prime}-\bar{\xi}\right)\right| \sim|\xi|^{\operatorname{Re}\left(a+a^{\prime}\right)-1}
$$

\subsection{Hypergeometric functions of the complex field}

Let $a_{1}\left|a_{1}^{\prime}, \ldots, a_{q}\right| a_{q}^{\prime}, b_{1}\left|b_{1}^{\prime}, \ldots, b_{p}\right| b_{p}^{\prime} \in \Lambda_{\mathbb{C}}$. Temporarily, we assume that they satisfy the conditions:

$$
\operatorname{Re}\left(a_{\alpha}+a_{\alpha}^{\prime}\right)>0, \quad \operatorname{Re}\left(b_{\beta}+b_{\beta}^{\prime}\right)>0 .
$$

and

$$
\sum_{\alpha} \operatorname{Re}\left(a_{\alpha}+a_{\alpha}^{\prime}\right)+\sum_{\beta} \operatorname{Re}\left(b_{\beta}+b_{\beta}^{\prime}\right)<p+q .
$$

We define the following function on $\Lambda_{\mathbb{C}}$ :

$$
\begin{aligned}
{ }_{p} \mathcal{K}_{q}^{\mathbb{C}}\left[\begin{array}{l}
\left(a \mid a^{\prime}\right) \\
\left(b \mid b^{\prime}\right)
\end{array} ; \frac{k+\sigma}{2} \mid \frac{-k+\sigma}{2}\right] \\
:=\prod_{\alpha=1}^{q} \Gamma^{\mathbb{C}}\left(a_{\alpha}+\frac{k+\sigma}{2} \mid a_{\alpha}^{\prime}+\frac{-k+\sigma}{2}\right) \prod_{\beta=1}^{p} \Gamma^{\mathbb{C}}\left(b_{\beta}+\frac{-k-\sigma}{2} \mid b_{\beta}^{\prime}+\frac{k-\sigma}{2}\right) .
\end{aligned}
$$

Next, we define the hypergeometric functions of the complex field as the contour integral:

$$
{ }_{p} G_{q}^{\mathbb{C}}\left[\begin{array}{l}
\left(a \mid a^{\prime}\right) \\
\left(b \mid b^{\prime}\right)
\end{array} ; z\right]:=\frac{1}{2 \pi \mathrm{i}} \sum_{k \in \mathbb{Z}} \int_{\mathrm{i} \mathbb{R}} p \mathcal{K}_{q}^{\mathbb{C}}\left[\begin{array}{l}
\left(a \mid a^{\prime}\right) \\
\left(b \mid b^{\prime}\right)
\end{array} ; \frac{k+\sigma}{2} \mid \frac{-k+\sigma}{2}\right] z^{\frac{-k-\sigma}{2} \mid \frac{k-\sigma}{2}} \mathrm{~d} \sigma .
$$

The integration is taken along the imaginary axis $i \mathbb{R}$. The condition (1.13) provides the conditional convergence of the integral in $\sigma$ and the absolute convergence of the series (see Sections 2.2 and 2.3 below). Under the stronger condition

$$
\sum_{\alpha} \operatorname{Re}\left(a_{\alpha}+a_{\alpha}^{\prime}\right)+\sum_{\beta} \operatorname{Re}\left(b_{\beta}+b_{\beta}^{\prime}\right)<p+q-1
$$

all integrals in $\sigma$ convergence absolutely (this follows from (1.11)).

The expression (1.14) has poles (they are analyzed in Section 2.3) in $\sigma$ originating from two groups of factors. We call poles of the factors $\Gamma^{\mathbb{C}}\left(a+\frac{k+\sigma}{2} \mid a^{\prime}+\frac{-k+\sigma}{2}\right)$ left poles, the poles of the factors $\Gamma^{\mathbb{C}}\left(b+\frac{-k-\sigma}{2} \mid b^{\prime}+\frac{k-\sigma}{2}\right)$ right poles. Under the conditions (1.12) left poles are contained in the left half-plane $\operatorname{Re} \sigma<0$, right poles are contained in the right half plane $\operatorname{Re} \sigma>0$, and the axis $i \mathbb{R}$ separates these groups of poles. 
As usual (see [43]) we can write the analytic continuation of ${ }_{p} G_{q}^{\mathbb{C}}\left[\begin{array}{c}\left(a \mid a^{\prime}\right) \\ \left(b \mid b^{\prime}\right)\end{array} ; z\right]$ to a wider domain of $\left(a \mid a^{\prime}\right),\left(b \mid b^{\prime}\right)$ by moving integration contours. Let us omit the condition (1.12) and fix $a_{\alpha} \mid a_{\alpha}^{\prime}$, $b_{\beta} \mid b_{\beta}^{\prime}$. Then $i \mathbb{R}$ separates left and right poles for all but a finite number of summands. Assume

$$
a_{\alpha}+b_{\beta} \notin \mathbb{Z}_{+} \quad \text { for all } \alpha, \beta \text {. }
$$

For each $k$ we choose a contour $L_{k}$ that coincides with i $\mathbb{R}$ at infinity and separates left and right poles of the corresponding summand, the result of the integration does not depend on a choice of $L_{k}$. Then we replace the expression (1.15) by

$$
\sum_{k} \int_{L_{k}}
$$

and get an expression of our integral in the domain defined by conditions (1.13), (1.17).

\subsection{The statements of the paper}

For the same $\left(a \mid a^{\prime}\right),\left(b \mid b^{\prime}\right)$ we define the expression $\Sigma_{+}^{\mathbb{C}}(z)$ by

$$
\begin{aligned}
\Sigma_{+}^{\mathbb{C}}\left[\begin{array}{c}
\left(a \mid a^{\prime}\right) \\
\left(b \mid b^{\prime}\right)
\end{array} ; z\right]:=2 \sum_{j=1}^{q} z^{a_{j} \mid a_{j}^{\prime}} \cdot \prod_{\beta} \Gamma^{\mathbb{C}}\left(b_{\beta}+a_{j} \mid b_{\beta}^{\prime}+a_{j}^{\prime}\right) \cdot \prod_{\alpha \neq j} \Gamma^{\mathbb{C}}\left(a_{\alpha}-a_{j} \mid a_{\alpha}^{\prime}-a_{j}^{\prime}\right) \\
\quad \times{ }_{p} F_{q-1}\left[\begin{array}{c}
\left(b_{\alpha}+a_{j}\right) \\
\left(1-a_{\alpha}+a_{j}\right) \backslash j
\end{array} ;(-1)^{q} z\right]{ }_{p} F_{q-1}\left[\begin{array}{c}
\left(b_{\alpha}^{\prime}+a_{j}^{\prime}\right) \\
\left(1-a_{\alpha}^{\prime}+a_{j}^{\prime}\right) \backslash j
\end{array} ;(-1)^{p} \bar{z}\right] .
\end{aligned}
$$

We also define the expression $\Sigma_{-}^{\mathbb{C}}(z)$ by

$$
\Sigma_{-}^{\mathbb{C}}\left[\begin{array}{c}
\left(a \mid a^{\prime}\right) \\
\left(b \mid b^{\prime}\right)
\end{array} ; z\right]:=\Sigma_{+}^{\mathbb{C}}\left[\begin{array}{l}
\left(b \mid b^{\prime}\right) \\
\left(a \mid a^{\prime}\right)
\end{array} ; z^{-1}\right] .
$$

Theorem 1.2. Let $\left(a \mid a^{\prime}\right),\left(b \mid b^{\prime}\right)$ satisfy the conditions (1.12) and (1.13).

(a) For $q>p$ we have

$$
{ }_{p} G_{q}^{\mathbb{C}}\left[\begin{array}{c}
\left(a \mid a^{\prime}\right) \\
\left(b \mid b^{\prime}\right)
\end{array} ; z\right]=\Sigma_{+}^{\mathbb{C}}\left[\begin{array}{c}
\left(a \mid a^{\prime}\right) \\
\left(b \mid b^{\prime}\right)
\end{array} ; z\right]
$$

and for $q<p$ we have ${ }_{p} G_{q}^{\mathbb{C}}(z)=\Sigma_{-}^{\mathbb{C}}(z)$.

(b) For $p=q$

$$
{ }_{p} G_{q}^{\mathbb{C}}(z)= \begin{cases}\Sigma_{+}^{\mathbb{C}}(z), & \text { if }|z|<1, \\ \Sigma_{-}^{\mathbb{C}}(z), & \text { if }|z|>1 .\end{cases}
$$

Remark 1.3. This statement and the main argument for its proof are potentially contained in the paper by Ismagilov [24, Lemma 2].

Corollary 1.4. The function $F(z):={ }_{p} G_{q}^{\mathbb{C}}\left[\begin{array}{c}\left(a \mid a^{\prime}\right) \\ \left(b \mid b^{\prime}\right)\end{array} ; z\right]$ satisfies the following system of differential equations

$$
\mathcal{D} F=0, \quad \overline{\mathcal{D}} F=0,
$$

where

$$
\begin{aligned}
& \mathcal{D}:=(-1)^{q} \prod_{\alpha=1}^{p}\left(z \frac{\partial}{\partial z}+a_{\alpha}\right)-z \prod_{\beta=1}^{q}\left(z \frac{\partial}{\partial z}-b_{\beta}\right), \\
& \overline{\mathcal{D}}:=(-1)^{p} \prod_{\alpha=1}^{p}\left(\bar{z} \frac{\partial}{\partial \bar{z}}+a_{\alpha}^{\prime}\right)-\bar{z} \prod_{\beta=1}^{q}\left(\bar{z} \frac{\partial}{\partial \bar{z}}-b_{\beta}^{\prime}\right) .
\end{aligned}
$$


Consider the expression ${ }_{p} G_{q}^{\mathbb{C}}\left[\begin{array}{c}\left(a \mid a^{\prime}\right) \\ \left(b \mid b^{\prime}\right)\end{array} ; z\right]$ as a function of a variable $z$ and parameters $\left(a \mid a^{\prime}\right)$, $\left(b \mid b^{\prime}\right)$, i.e., a function defined on a certain domain in the space

$$
\mathbb{C} \times\left(\Lambda_{\mathbb{C}}\right)^{p+q} \simeq \mathbb{C} \times \mathbb{Z}^{p+q} \times \mathbb{C}^{p+q} .
$$

Fixing integer parameters ${ }^{3}\left(a-a^{\prime}\right),\left(b-b^{\prime}\right)$ we get a countable collection of functions on domains in $\mathbb{C} \times \mathbb{C}^{p+q}$.

Next, fix $\alpha$, $\beta$, fix also $m, m^{\prime} \in \mathbb{Z}_{+}$. Consider the subset $S\left(\alpha, \beta ; m, m^{\prime}\right)$ in $\mathbb{C} \times\left(\Lambda_{\mathbb{C}}\right)^{p+q}$ defined by the equations

$$
\left\{\begin{array}{l}
a_{\alpha}+b_{\beta}=m, \\
a_{\alpha}^{\prime}+b_{\beta}^{\prime}=m^{\prime} .
\end{array}\right.
$$

Proposition 1.5. For fixed $\left(a-a^{\prime}\right) \in \mathbb{Z}^{p+q},\left(b-b^{\prime}\right) \in \mathbb{Z}^{p+q}$ the expression ${ }_{p} G_{q}^{\mathbb{C}}\left[\begin{array}{c}\left(a \mid a^{\prime}\right) \\ \left(b \mid b^{\prime}\right)\end{array} ; z\right]$ as a function of $(a),(b), z$ admits an extension to a function which is real analytic in $z$ (i.e., real analytic as a function in two variables $\operatorname{Re} z, \operatorname{Im} z)$ in the domain

$$
\begin{aligned}
& z \in \mathbb{C} \backslash\{0\}, \quad \text { if } p \neq q, \\
& z \in \mathbb{C} \backslash\left\{0,(-1)^{p}\right\}, \quad \text { if } p=q,
\end{aligned}
$$

and meromorphic in $(a)$, (b) with singularities (simple poles) located in

$$
\bigcup_{\alpha, \beta, m, m^{\prime}} S\left(\alpha, \beta ; m, m^{\prime}\right) \text {. }
$$

\section{Lemma 1.6.}

(a) Let the parameters $\left(a \mid a^{\prime}\right),\left(b \mid b^{\prime}\right)$ satisfy the conditions

$$
\begin{aligned}
& \operatorname{Re}\left(a_{\alpha}+a_{\alpha}^{\prime}\right)>0, \quad \operatorname{Re}\left(b_{\beta}+b_{\beta}^{\prime}\right)>0, \\
& \sum_{\alpha} \operatorname{Re}\left(a_{\alpha}+a_{\alpha}^{\prime}\right)+\sum_{\beta}\left(b_{\beta}+b_{\beta}^{\prime}\right)<p+q-1 .
\end{aligned}
$$

Then the function ${ }_{p} G_{q}^{\mathbb{C}}\left[\begin{array}{c}\left(a \mid a^{\prime}\right) \\ \left(b \mid b^{\prime}\right)\end{array} ; z\right]$ is contained in $L^{2}\left(\mathbb{C},|z|^{-2} \mathrm{~d} \overline{\bar{z}}\right)$.

(b) Under the same conditions

$$
\frac{1}{2 \pi} \int_{\mathbb{C}} z^{\sigma-1 \mid \sigma^{\prime}-1}{ }_{p} G_{q}^{\mathbb{C}}\left[\begin{array}{c}
\left(a \mid a^{\prime}\right) \\
\left(b \mid b^{\prime}\right)
\end{array} ; z\right] \mathrm{d} \overline{\bar{z}}={ }_{p} \mathcal{K}_{q}^{\mathbb{C}}\left[\begin{array}{c}
\left(a \mid a^{\prime}\right) \\
\left(b \mid b^{\prime}\right)
\end{array} ; \sigma \mid \sigma^{\prime}\right],
$$

where the integral is understood as a Mellin transform in $L^{2}$.

Theorem 1.7. Let two functions

$$
{ }_{p} G_{q}^{\mathbb{C}}\left[\begin{array}{l}
\left(a \mid a^{\prime}\right) \\
\left(b \mid b^{\prime}\right)
\end{array} ; z\right], \quad{ }_{r} G_{s}^{\mathbb{C}}\left[\begin{array}{l}
\left(c \mid c^{\prime}\right) \\
\left(d \mid d^{\prime}\right)
\end{array} ; z\right]
$$

be contained in $L^{2}\left(\mathbb{C},|z|^{-2} \mathrm{~d} \overline{\bar{z}}\right)$. Then

$$
\frac{1}{2 \pi} \int_{\mathbb{C}}{ }_{p} G_{q}^{\mathbb{C}}\left[\begin{array}{l}
\left(a \mid a^{\prime}\right) \\
\left(b \mid b^{\prime}\right)
\end{array} ; z\right] \cdot{ }_{r} G_{s}^{\mathbb{C}}\left[\begin{array}{l}
\left(c \mid c^{\prime}\right) \\
\left(d \mid d^{\prime}\right)
\end{array} ; \frac{t}{z}\right] \frac{\mathrm{d} \overline{\bar{z}}}{z^{1 \mid 1}}={ }_{p+r} G_{q+s}^{\mathbb{C}}\left[\begin{array}{l}
\left(a \mid a^{\prime}\right),\left(c \mid c^{\prime}\right) \\
\left(b \mid b^{\prime}\right),\left(d \mid d^{\prime}\right)
\end{array} ; t\right] .
$$

Taking $p=q=1$ (see (3.2)) we get a counterpart of formula (1.3):

$$
\begin{array}{r}
\frac{1}{2 \pi} \int_{\mathbb{C}} z^{a \mid a^{\prime}}(1+z)^{-a-b \mid-a^{\prime}-b^{\prime}} \cdot{ }_{r} G_{s}^{\mathbb{C}}\left[\begin{array}{l}
\left(c \mid c^{\prime}\right) \\
\left(d \mid d^{\prime}\right)
\end{array} ; \frac{t}{z}\right] \frac{\mathrm{d} \overline{\bar{z}}}{z^{1 \mid 1}} \\
\quad=\frac{1}{2 \Gamma^{\mathbb{C}}\left(a+b \mid a^{\prime}+b^{\prime}\right)} 1+r G_{1+s}^{\mathbb{C}}\left[\begin{array}{l}
a \mid a^{\prime},\left(c \mid c^{\prime}\right) \\
b \mid b^{\prime},\left(d \mid d^{\prime}\right)
\end{array} ; t\right] .
\end{array}
$$

\footnotetext{
${ }^{3}$ The conditions (1.12) and (1.13) allow arbitrary integer parameters.
} 


\section{Proofs}

\subsection{Asymptotics of $\Gamma^{\mathbb{C}}$}

Let us derive formula (1.10) for the asymptotics of the function $\Gamma^{C}\left(a+\xi \mid a^{\prime}-\bar{\xi}\right)$. First, let us verify that the right-hand side is single-valued on $\Lambda$. We must verify that the expression

$$
\exp \{\xi \ln \xi-\bar{\xi} \ln \bar{\xi}\}, \quad \xi=\frac{k+\mathrm{i} s}{2}
$$

is single valued. Represent $\xi=r \mathrm{e}^{\mathrm{i} \varphi}$. We transform our expression as

$$
\begin{gathered}
\exp \{\xi(\ln (r)+\mathrm{i} \varphi+2 \pi \mathrm{i} n)-\bar{\xi}(\ln (r)-\mathrm{i} \varphi-2 \pi \mathrm{i} n)\} \\
=\exp \{(\xi-\bar{\xi}) \ln r+(\xi+\bar{\xi})(\mathrm{i} \varphi+2 \pi \mathrm{i} n)\}
\end{gathered}
$$

We have $\xi+\bar{\xi} \in \mathbb{Z}$ and therefore the exponential is single valued.

Next, we apply the Stirling formula in the form (see [13, equation (1.18(3))])

$$
\ln \Gamma(c+z)=\left(z+c-\frac{1}{2}\right) \ln z-z+\frac{1}{2} \ln (2 \pi)+O\left(z^{-1}\right), \quad \text { where }|\arg (z)|<\pi-\varepsilon,
$$

and get

$$
\begin{aligned}
& \Gamma(a+\xi) \simeq \sqrt{2 \pi} \exp \left\{\left(\xi+a-\frac{1}{2}\right) \ln \xi-\xi\right\}, \\
& \Gamma\left(1-a^{\prime}-\bar{\xi}\right) \simeq \sqrt{2 \pi} \exp \left\{\left(\bar{\xi}-a^{\prime}+\frac{1}{2}\right) \ln \bar{\xi}-\bar{\xi}\right\} .
\end{aligned}
$$

The ratio gives us (1.10). An evaluation of the asymptotics in the sector $|\arg (-z)|<\pi-\varepsilon$ gives the same result.

Corollary 2.1. Let $\operatorname{Re}\left(a_{\alpha}+a_{\alpha}^{\prime}\right)>0, \operatorname{Re}\left(b_{\beta}+b_{\beta}^{\prime}\right)>0$. Denote

$$
v:=\sum\left(a_{\alpha}+a_{\alpha}^{\prime}\right)+\sum\left(b_{\beta}+b_{\beta}^{\prime}\right) .
$$

(a) If $v<p+q$, then $F(k$, is $):={ }_{p} \mathcal{K}_{q}^{\mathbb{C}}\left[\begin{array}{l}\left(a \mid a^{\prime}\right) \\ \left(b \mid b^{\prime}\right)\end{array} ; \frac{k+\mathrm{i} s}{2} \mid ; \frac{-k+\mathrm{i} s}{2}\right]$ tends to 0 as $|k+\mathrm{i} s|$ tends to $\infty$.

(b) If $v<p+q-1$, then for each $k$ the function $F(k$, is $)$ as a function in $s$ is integrable. Under the same condition $F(k$, is $)$ is contained in $L^{2}(\Lambda)$.

(c) If $v<p+q-2$, then $F\left(k\right.$, is) is contained in $L^{1}(\Lambda)$.

Remark 2.2. Formula (1.10) also gives us asymptotics of ${ }_{p} \mathcal{K}_{q}^{\mathbb{C}}$ on vertical lines $\sigma=h+\mathrm{i} s$. Indeed,

$$
\Gamma\left(a+\frac{k+h+\mathrm{i} s}{2} \mid a^{\prime}+\frac{-k+h+\mathrm{i} s}{2}\right)=\Gamma\left(a+\frac{h}{2}+\frac{k+\mathrm{i} s}{2} \mid a^{\prime}+\frac{h}{2}+\frac{-k+\mathrm{i} s}{2}\right),
$$

and we can control integrability under shifts of the integration contour.

\subsection{Decomposition of Mellin-Barnes integrals in residues}

Now we start to prove Theorem 1.2, i.e., to derive the quadratic expressions of the functions ${ }_{p} G_{q}^{\mathbb{C}}$ in terms of the usual hypergeometric functions ${ }_{p} F_{q}$.

First, we write the definition (1.15) of ${ }_{p} G_{q}^{\mathbb{C}}$ in the form

$$
\sum_{k \in \mathbb{Z}} z^{-k / 2 \mid k / 2} I_{k}(z), \quad I_{k}(z)=\frac{1}{2 \pi \mathrm{i}} \int(\ldots)|z|^{-\sigma} \mathrm{d} \sigma .
$$


The integrals $I_{k}(z)$ are special cases of Mellin-Barnes integrals, i.e., integrals of the type

$$
J_{C, D}^{A, B}(a, b, c, d ; u)=\frac{1}{2 \pi \mathrm{i}} \int_{L} \frac{\prod_{\alpha=1}^{A} \Gamma\left(a_{\alpha}+\sigma\right) \prod_{\beta=1}^{B} \Gamma\left(b_{\beta}-\sigma\right)}{\prod_{\gamma=1}^{C} \Gamma\left(c_{\gamma}+\sigma\right) \prod_{\delta=1}^{D} \Gamma\left(d_{\delta}-\sigma\right)} u^{-s} \mathrm{~d} s,
$$

where $L$ is a contour separating poles of factors $\Gamma\left(a_{\alpha}+\sigma\right)$ and poles of $\Gamma\left(b_{\beta}-\sigma\right)$. The behavior of such Mellin-Barnes integrals (Meijer $G$-functions) was investigated by Meijer, his results are exposed in $[3,30,31]$. Under certain conditions $J(a, b, c, d ; u)$ can be expressed in terms of functions $\Sigma_{ \pm}(z)$, where $\Sigma_{+}(z)$ is the sum of residues of the integrand at poles of factors $\Gamma\left(a_{\alpha}+\sigma\right)$, and $\Sigma_{-}(z)$ the sum of residues at poles of $\Gamma\left(b_{\beta}-\sigma\right)$.

In our case $A=D=q, B=C=p$, and $u=|z|$ is a positive real. The contour $L$ coincides with the imaginary axis at infinity. The asymptotics of the absolute value of the integrand on the imaginary axis is

$$
\sim|\sigma|^{\sum_{\alpha=1}^{q} \operatorname{Re}\left(a_{\alpha}-d_{\alpha}\right)+\sum_{\beta=1}^{p} \operatorname{Re}\left(b_{\beta}-c_{\beta}\right)},
$$

see [13, equation (1.18(4))]. One of the statements of [31, Theorem 18] implies that if the integrand tends to zero on the imaginary axis, then the integral $J(a, b, c, d ; u)$ conditionally converges if the integrand tends to 0 at infinity for $u>0$ and is given by

$-\Sigma_{+}(u)$ for $q>p$;

$-\Sigma_{-}(u)$ for $q<p$;

- for $p=q$ we have $\Sigma_{+}(u)$ if $0<u<1$ and $\Sigma_{-}(u)$ for $u>1$.

If $\sum \operatorname{Re}\left(a_{\alpha}-d_{\alpha}\right)+\sum \operatorname{Re}\left(b_{\beta}-c_{\beta}\right)<-1$, then the absolute convergence is obvious.

In our case $a_{\alpha}$ is replaced by $a_{\alpha}+k / 2, b_{\beta}$ by $b_{\beta}-k / 2$, and $c_{\beta} \mapsto 1-b_{\beta}^{\prime}-k / 2, d_{\alpha} \mapsto 1-a_{\alpha}^{\prime}+k / 2$. Therefore under our conditions (1.12), (1.13) all integrals $I_{k}$ converge.

\subsection{Evaluation of sums of residues}

Lemma 2.3. Fix $a \mid a^{\prime}$ and consider the following family of functions:

$$
\gamma_{k}(\sigma):=\Gamma^{\mathbb{C}}\left(a+\frac{k+\sigma}{2} \mid a^{\prime}+\frac{-k+\sigma}{2}\right) .
$$

Denote by $\Omega$ the sets of all points $(k, \sigma) \in \mathbb{Z} \times \mathbb{C}$ such that $\sigma$ is a pole of $\gamma_{k}(\sigma)$.

(a) The set $\Omega$ is contained in the half-plane $\operatorname{Re} \sigma<0$ if and only if $\operatorname{Re}\left(a+a^{\prime}\right)>0$.

(b) The set $\Omega$ consists of points

$$
\begin{aligned}
k & =-m+m^{\prime}-a+a^{\prime}, \\
\sigma & =-m-m^{\prime}-a-a^{\prime},
\end{aligned}
$$

where $m, m^{\prime}$ range in $\mathbb{Z}_{+}$.

Proof. (a) Let all poles be contained in the domain $\operatorname{Re} \sigma<0$. Taking $k=a^{\prime}-a$ we get

$$
\gamma_{a^{\prime}-a}(\sigma)=\Gamma^{\mathbb{C}}\left(a+\frac{a^{\prime}-a+\sigma}{2} \mid a^{\prime}+\frac{-a^{\prime}+a+\sigma}{2}\right)=\Gamma^{\mathbb{C}}\left(\frac{a+a^{\prime}}{2}+\frac{\sigma}{2} \mid \frac{a+a^{\prime}}{2}+\frac{\sigma}{2}\right) .
$$

The point $\sigma=-\left(a+a^{\prime}\right)$ is a pole of $\gamma_{a^{\prime}-a}(\sigma)$. Therefore, $\operatorname{Re}\left(a+a^{\prime}\right)>0$.

Conversely, let $\operatorname{Re}\left(a+a^{\prime}\right)>0$. Consider $(k, \sigma) \in \Omega$. Then (see Section 1.3)

$$
a+\frac{k+\sigma}{2}=-m \in \mathbb{Z}_{-}, \quad a^{\prime}+\frac{-k+\sigma}{2}=-m^{\prime} \in \mathbb{Z}_{-} .
$$

Therefore $a+a^{\prime}+\sigma \in \mathbb{Z}_{-}$and $\operatorname{Im} \sigma<0$.

(b) We solve the system (2.2). 
Evaluation of the sum of residues (the proof of Theorem 1.2). For definiteness assume that $p \leqslant q$. Let us write the residue $R\left(j ; m, m^{\prime}\right)$ of the integrand (1.15) at a point

$$
k=-m+m^{\prime}-a_{j}+a_{j}^{\prime}, \quad \sigma=-m-m^{\prime}-a_{j}-a_{j}^{\prime} .
$$

We have

$$
\frac{k+\sigma}{2}=-a_{j}-m, \quad \frac{-k+\sigma}{2}=-a_{j}^{\prime}-m^{\prime},
$$

and keeping in mind (1.9) we get

$$
\begin{aligned}
R\left(j ; m, m^{\prime}\right)= & \frac{2(-1)^{m}}{m ! m^{\prime} !} \prod_{\alpha \neq j} \Gamma^{\mathbb{C}}\left(a_{\alpha}-a_{j}-m \mid a_{\alpha}^{\prime}-a_{j}^{\prime}-m^{\prime}\right) \\
& \times \prod_{\beta} \Gamma^{\mathbb{C}}\left(b_{\beta}+a_{j}+m \mid b_{\beta}^{\prime}+a_{j}^{\prime}+m^{\prime}\right) \times z^{a_{j}+m \mid a_{j}^{\prime}+m^{\prime}} .
\end{aligned}
$$

Applying (1.8c) and (1.8d) we come to

$$
\begin{aligned}
R\left(j ; m, m^{\prime}\right)= & 2 \frac{(-1)^{m}}{m ! m^{\prime} !} \prod_{\alpha \neq j} \frac{\Gamma^{\mathbb{C}}\left(a_{\alpha}-a_{j} \mid a_{\alpha}^{\prime}-a_{j}^{\prime}\right)(-1)^{m}}{\left(1-a_{\alpha}+a_{j}\right)_{m}\left(1-a_{\alpha}^{\prime}+a_{j}^{\prime}\right)_{m^{\prime}}} \\
& \left.\times \prod_{\beta} \Gamma^{\mathbb{C}}\left(b_{\beta}+a_{j} \mid b_{\beta}^{\prime}+a_{j}^{\prime}\right) b_{\beta}+a_{j}\right)_{m}\left(b_{\beta}^{\prime}+a_{j}^{\prime}\right)_{m^{\prime}}(-1)^{m^{\prime}} \times z^{a_{j}+m \mid a_{j}^{\prime}+m^{\prime}} .
\end{aligned}
$$

Reordering factors, we get

$$
\begin{aligned}
& 2 z^{a_{j} \mid a_{j}^{\prime}} \prod_{\alpha \neq j} \Gamma^{\mathbb{C}}\left(a_{\alpha}-a_{j} \mid a_{\alpha}^{\prime}-a_{j}^{\prime}\right) \prod_{\beta} \Gamma^{\mathbb{C}}\left(b_{\beta}+a_{j} \mid b_{\beta}^{\prime}+a_{j}^{\prime}\right) \\
& \quad \times(-1)^{m q} \frac{z^{m} \prod_{\beta}\left(b_{\beta}+a_{j}\right)_{m}}{m ! \prod_{\alpha \neq j}\left(1-a_{\alpha}+a_{j}\right)_{m}} \times(-1)^{m^{\prime} p} \frac{\bar{z}^{m^{\prime}} \prod_{\beta}\left(b_{\beta}^{\prime}+a_{j}^{\prime}\right)_{m^{\prime}}}{m^{\prime} ! \prod_{\alpha \neq j}\left(1-a_{\alpha}^{\prime}+a_{j}^{\prime}\right)_{m^{\prime}}} .
\end{aligned}
$$

Formal calculation with series gives

$$
\begin{aligned}
\sum_{m, m^{\prime}} R\left(j, m, m^{\prime}\right)= & 2 z^{a_{j} \mid a_{j}^{\prime}} \prod_{\alpha \neq j} \Gamma^{\mathbb{C}}\left(a_{\alpha}-a_{j} \mid a_{\alpha}^{\prime}-a_{j}^{\prime}\right) \prod_{\beta} \Gamma^{\mathbb{C}}\left(b_{\beta}+a_{j} \mid b_{\beta}^{\prime}+a_{j}^{\prime}\right) \\
& \times \sum_{m} \frac{\left((-1)^{q} z\right)^{m} \prod_{\beta}\left(b_{\beta}+a_{j}\right)_{m}}{m ! \prod_{\alpha \neq j}\left(1-a_{\alpha}+a_{j}\right)_{m}} \times \sum_{m^{\prime}} \frac{\left((-1)^{p} \bar{z}\right)^{m^{\prime}} \prod_{\beta}\left(b_{\beta}+a_{j}\right)_{m^{\prime}}}{m^{\prime} ! \prod_{\alpha \neq j}\left(1-a_{\alpha}^{\prime}+a_{j}^{\prime}\right)_{m^{\prime}}} .
\end{aligned}
$$

We get a product of two hypergeometric series whose radius of convergence is $\infty$ if $q>p$ and 1 if $q=p$. They are absolutely convergent in the disk of convergence, therefore the last identity really takes place. Therefore

$$
\sum_{j} \sum_{m, m^{\prime}} R\left(j, m, m^{\prime}\right)=\Sigma_{+}^{\mathbb{C}}(z) .
$$

On the other hand the absolute convergence allows us to write

$$
\sum_{m, m^{\prime}} R\left(j, m, m^{\prime}\right)=\sum_{k \in \mathbb{Z}} \sum_{m, m^{\prime}: m-m^{\prime}=k} R\left(j, m, m^{\prime}\right)=\sum_{k \in \mathbb{Z}}(z / \bar{z})^{k / 2} I_{k}
$$

Thus we proved the coincidence of ${ }_{p} G_{q}^{\mathbb{C}}$ and $\Sigma_{+}^{\mathbb{C}}$. The summation of residues at the right poles of ${ }_{p} \mathcal{K}_{q}^{\mathbb{C}}$ is similar.

It is important that the convergence of the series $\sum_{k \in \mathbb{Z}}$ in (2.3) is locally uniform in the parameters $a_{\alpha}, b_{\beta}$ near any point $\left(a_{0}\right) \in \mathbb{C}^{q},\left(b_{0}\right) \in \mathbb{C}^{p}$, for which the coefficients at $z^{l} \bar{z}^{\prime}$ are well-defined. 


\subsection{The application of the Mellin transform}

Our next purpose is Theorem 1.7 about evaluations of the convolution integrals

$$
\int_{\mathbb{C}}{ }_{p} G_{q}^{\mathbb{C}}(\ldots ; z){ }_{r} G_{s}^{\mathbb{C}}(\ldots ; t / z) \frac{\mathrm{d} \overline{\bar{z}}}{z^{1 \mid 1}} .
$$

Under the condition of Corollary 2.1 the integral (1.15) defining a ${ }_{p} G_{q}^{\mathbb{C}}$-function is the inverse Mellin transform of the function ${ }_{p} \mathcal{K}_{q}^{\mathbb{C}}$. Therefore the function ${ }_{p} \mathcal{K}_{q}^{\mathbb{C}}$ is the direct Mellin transform of ${ }_{p} G_{q}^{\mathbb{C}}$. This is the statement of Lemma 1.6.

The Mellin transform is the Fourier transform on the group $\mathbb{C}^{\times}$, therefore it sends multiplicative convolutions to products. This property remains to be valid if both functions are contained in $L^{2}$, see [44, Theorem 64, Section 3.13]. ${ }^{4}$ This implies Theorem 1.7.

\subsection{The differential equations}

Here we derive the system (1.19) of differential equations for a function ${ }_{p} G_{q}^{\mathbb{C}}$ (the statement of Corollary 1.4). We can use the explicit expressions $\Sigma_{ \pm}^{\mathbb{C}}$ for ${ }_{p} G_{q}^{\mathbb{C}}$ obtained in Theorem 1.2. The formula (1.18) for $\Sigma_{+}^{\mathbb{C}}$ has the form

$$
\sum C_{j} \Phi_{j}(z) \cdot \Psi_{j}(\bar{z})
$$

where $C_{j}$ do not depend on $z$ and

$$
\Phi_{j}(z)=z^{a_{j}} F_{q-1}\left[\begin{array}{c}
\left(b_{\alpha}+a_{j}\right) \\
\left(1-a_{\alpha}+a_{j}\right) \backslash j
\end{array} ;(-1)^{p+q} z\right] .
$$

The equation $\mathcal{D} F=0$ of the system (1.19) is slightly modified equation for generalized hypergeometric functions, see [43, Section 2.1.2] or [30, Section 5.2]. The functions $\Phi_{j}(z)$ form a fundamental system of solutions of this equation in holomorphic functions on $\mathbb{C}$.

This implies Corollary 1.4.

Remark 2.4. The statement also follows from the identities (3.10a)-(3.11b) established below.

\subsection{The analytic continuation}

It remains to prove Proposition 1.5 about the analytic continuation of the function ${ }_{p} G_{q}^{\mathbb{C}}\left[\begin{array}{c}\left(a \mid a^{\prime}\right) \\ \left(b \mid b^{\prime}\right)\end{array} ; z\right]$ in the variable $z$ and in the parameters $\left(a \mid a^{\prime}\right),\left(b \mid b^{\prime}\right)$.

(1). Assume $p \neq q$, for definiteness let $p<q$. First, we omit the condition (1.12). A value of ${ }_{p} G_{q}^{\mathbb{C}}$ at $z$ is defined by an infinite sum of contour integrals $I_{k}$ and the convergence is locally uniform in the parameters. For all but a finite number of summands the imaginary axis iR separates the left poles and right poles of the integrand (see the terminology of Section 1.4). Consider one of the remaining summands, say $I_{m}$. A contour $L_{m}$ separating left poles and right poles of (1.15) exists if and only if left poles differ from right poles. The condition (1.21) is the condition of collision of left and right poles. Two separation contours $L_{m}, L_{m}^{\prime}$ can be nonhomotopic. However

$$
\frac{1}{2 \pi \mathrm{i}}\left(\int_{L_{m}}-\int_{\mathrm{i} \mathbb{R}}\right)
$$

\footnotetext{
${ }^{4}$ Proof. Denote by $C_{0}$ the space of continuous functions that have zero limit at infinity. The product is a continuous operation $L^{2} \times L^{2} \rightarrow L^{1}$. The Fourier transform sends $L^{2} \rightarrow L^{2}$ bijectively, and sends $L^{1} \rightarrow C_{0}$. The convolution is a continuous operation $L^{2} \times L^{2} \rightarrow C_{0}$. Therefore the Fourier transform sends a product of two $L^{2}$-functions to the convolution.
} 
is a sum of residues at right poles contained in the half-plane $\operatorname{Re} \sigma<0$ minus a sum of residues at left poles contained in the half-plane $\operatorname{Re} \sigma>0$. This expression does not depend on a choice of separation contour. Clearly, a contour integral is holomorphic in the parameters.

So the sum of contour integrals is well-defined in the domain defined by conditions (1.16), (1.17) (absolute convergence and absence of collisions).

On the other hand the generalized hypergeometric functions ${ }_{p} F_{q-1}$ meromorphically depend on their parameters, and therefore $\Sigma_{+}(z)$ meromorphically depends on parameters and this allows to omit the condition (1.13) for the convergence. Sums of power series are real analytic and this implies real analyticity of $\Sigma_{+}(z)$ for $z \in \mathbb{C} \backslash 0$.

Possible poles are located at hyperplanes

$$
\left\{\begin{array}{l}
a_{j}+b_{\beta} \in \mathbb{Z}_{-}, \\
a_{j}^{\prime}+b_{\beta}^{\prime} \in \mathbb{Z}_{-},
\end{array}\right.
$$

for some $j$ and $\beta$ and

$$
\left\{\begin{array}{l}
a_{\alpha}-a_{j} \in \mathbb{Z}, \\
a_{\alpha}^{\prime}-a_{j}^{\prime} \in \mathbb{Z},
\end{array}\right.
$$

for some $j, \alpha$. It remains to notice that singularities of the second type are removable under the open condition (1.16) and therefore they are always removable.

(2). Let $p=q$. The same argument can be applied for $|z|<1$ and $|z|>1$, it remains to examine the function on the circle $|z|=1$. We extend ${ }_{p} G_{q}^{\mathbb{C}}(z)$ to the same domain of parameters as above and come away to a smaller domain ${ }^{5}$

$$
\sum \operatorname{Re}\left(a_{\alpha}+a_{\alpha}^{\prime}\right)+\sum \operatorname{Re}\left(b_{\beta}+b_{\beta}^{\prime}\right)<p-2 .
$$

Then the contour integral in (1.15) has continuous partial derivatives up to order $p$. By continuity the equation $\mathcal{D} F=0$ is valid on the circle $|z|=1$. The operator $\mathcal{D}$ is elliptic for $z \neq 0, \pm 1$. Therefore under the condition (2.4) solutions of the equation $\mathcal{D} F=0$ are analytic (see, e.g., [22, Theorem 8.5.1]). Consider a point $z_{0} \neq 1$ on the circle $|z|=1$. Any generalized hypergeometric function defined in the disk $|z|<1$ has an analytic continuation to a neighborhood $U$ of $z_{0}$. Therefore the expression (1.18) for ${ }_{p} G_{q}^{\mathbb{C}}(z)$ provides us an analytic continuation of $\Sigma_{+}(z)$ to $U$ (and coincides with $\Sigma_{-}^{\mathbb{C}}$ in the intersection of $U$ and the domain $|z|>1$ ). This expression is meromorphic in $(a),(b)$ as above.

\section{Additions}

\subsection{Some simple cases}

\subsubsection{The exponential}

For $p=0, q=1$ formula (1.18) gives

$$
{ }_{0} G_{1}^{\mathbb{C}}\left[\begin{array}{c}
a \mid a^{\prime} \\
-
\end{array} z\right]=2 z^{a \mid a^{\prime}} \mathrm{e}^{-z} \mathrm{e}^{\bar{z}}
$$

\footnotetext{
${ }^{5}$ So we assume $p>2$. For the case $p=2$ the analyticity on the circle is clear from explicit formulas, see [33]; the case $p=1$ is trivial, see (3.2) below.
} 


\subsubsection{The power function}

For $p=q=1$ we get

$$
\begin{aligned}
{ }_{1} G_{1}^{\mathbb{C}}\left[\begin{array}{l}
a \mid a^{\prime} \\
b \mid b^{\prime}
\end{array} ; z\right] & =2 z^{a \mid a^{\prime}} \Gamma^{\mathbb{C}}\left(a+b \mid a^{\prime}+b^{\prime}\right){ }_{1} F_{0}\left[\begin{array}{c}
a+b \\
-
\end{array}-z\right] \cdot{ }_{1} F_{0}\left[\begin{array}{c}
a^{\prime}+b^{\prime} \\
-
\end{array}-\bar{z}\right] \\
& =2 \Gamma^{\mathbb{C}}\left(a+b \mid a^{\prime}+b^{\prime}\right) z^{a \mid a^{\prime}}(1+z)^{-a-b \mid-a^{\prime}-b^{\prime}} .
\end{aligned}
$$

The series ${ }_{1} F_{0}$ converge in the disc $|z|<1$, but the final expression is well defined in $\mathbb{C} \backslash\{0,-1\}$.

\subsubsection{The Kummer functions}

For $p=1, q=2$ we get

$$
\begin{aligned}
& { }_{1} G_{2}^{\mathbb{C}}\left[\begin{array}{c}
a_{1}\left|a_{1}^{\prime}, a_{2}\right| a_{2}^{\prime} ; z \\
b \mid b^{\prime}
\end{array}\right]=2 \Gamma^{\mathbb{C}}\left(a_{1}+b \mid a_{1}^{\prime}+b\right) \Gamma^{\mathbb{C}}\left(a_{2}-a_{1} \mid a_{2}^{\prime}-a_{1}^{\prime}\right) \\
& \times z^{a_{1}}{ }_{1} F_{1}\left[\begin{array}{c}
a_{1}+b \\
1-a_{2}+a_{1}
\end{array} ; z\right] \bar{z}^{a_{1}^{\prime}}{ }_{1} F_{1}\left[\begin{array}{c}
a_{1}^{\prime}+b^{\prime} \\
1-a_{2}^{\prime}+a_{1}^{\prime}
\end{array} ;-\bar{z}\right] \\
& +\left\{\text { similar term obtained by the transposition } a_{1}\left|a_{1}^{\prime} \longleftrightarrow a_{2}\right| a_{2}^{\prime}\right\} \text {. }
\end{aligned}
$$

Denote the hypergeometric functions in these expression by $\Phi_{1}(z), \Phi_{1}^{\prime}(\bar{z}), \Phi_{2}(z), \Phi_{2}^{\prime}(\bar{z})$. Then $z^{a_{1}} \Phi_{1}(z), z^{a_{2}} \Phi_{2}(z)$ is a fundamental system of holomorphic solutions of the equation $\mathcal{D} F=0$ of the system (1.19), and $\bar{z}^{a_{1}^{\prime}} \Phi_{1}^{\prime}(\bar{z}), \bar{z}^{a_{2}^{\prime}} \Phi_{2}^{\prime}(\bar{z})$ is a fundamental system of antiholomorphic solutions of the equation $\overline{\mathcal{D}} F=0$ (generally, all these functions are ramified at 0 and $\infty$ ). Then the functions

$$
z^{a_{1}} \Phi_{1}(z) \bar{z}^{a_{1}^{\prime}} \Phi_{1}^{\prime}(\bar{z}), \quad z^{a_{2}} \Phi_{2}(z) \bar{z}^{a_{1}^{\prime}} \Phi_{1}^{\prime}(\bar{z}), \quad z^{a_{1}} \Phi_{1}(z) \bar{z}^{a_{2}^{\prime}} \Phi_{2}^{\prime}(\bar{z}), \quad z^{a_{2}} \Phi_{2}(z) \bar{z}^{a_{2}^{\prime}} \Phi_{2}^{\prime}(\bar{z})
$$

is a basis of the space of solutions of the system (1.19) in a neighborhood of any point $z_{0} \neq 0$, see [33, Proposition 3.8]. For $a_{1}-a_{2} \notin \mathbb{Z}$ solutions non-ramified at 0 and $\infty$ have the form

$$
C_{1} z^{a_{1} \mid a_{1}^{\prime}} \Phi_{1}(z) \Phi_{1}^{\prime}(\bar{z})+C_{2} z^{a_{2} \mid a_{2}^{\prime}} \Phi_{2}(z) \Phi_{2}^{\prime}(\bar{z}) .
$$

The asymptotic expansion of confluent hypergeometric function ${ }_{1} F_{1}(z)$ as $z \rightarrow \infty$ (see, e.g., [30, Section 4.7]) in the sector $|\arg z|<\pi-\varepsilon$ is

$$
\begin{aligned}
{ }_{1} F_{1}\left[\begin{array}{l}
a \\
b
\end{array} ; z\right]= & \frac{\Gamma(b)}{\Gamma(b-a)} \mathrm{e}^{\mathrm{i} \pi \operatorname{sgn}(\operatorname{Im} a)} z^{-a}\left(\sum_{n=0}^{K-1} \frac{(a)_{n}(1+a-b)_{n}}{n !}(-z)^{-n}+O\left(z^{-K}\right)\right) \\
& +\frac{\Gamma(b)}{\Gamma(a)} e^{z} z^{a-b}\left(\sum_{n=0}^{L-1} \frac{(b-a)_{n}(1-a)_{n}}{n !} z^{-n}+O\left(z^{-L}\right)\right) .
\end{aligned}
$$

For generic $C_{1}, C_{2}$ the growth of expression (3.4) as $|\operatorname{Re} z| \rightarrow \infty$ is exponential. For the linear combination (3.3) all exponential terms of the asymptotics disappear (and ratio of coefficients in (3.3) is uniquely defined by this condition, existence of such ratio a priori is non-obvious). A reminder is $O\left(z^{-N} \mathrm{e}^{z}\right)+O\left(z^{-N} \mathrm{e}^{-z}\right)+O(1)$ for any $N$, is too rough. However, under the conditions $\operatorname{Re}\left(a_{1}+a_{1}^{\prime}\right)>0, \operatorname{Re}\left(a_{2}+a_{2}^{\prime}\right)>0, \operatorname{Re}\left(a_{1}+a_{1}^{\prime}+a_{2}+a_{2}^{\prime}\right)<1$ our function ${ }_{2} G_{1}^{\mathbb{C}}$ is contained in $L^{2}\left(\mathbb{C},|z|^{-2} \mathrm{~d} \overline{\bar{z}}\right)$.

\subsubsection{The Bessel functions}

For $p=0, q=2$ we get an expression

$$
\begin{aligned}
& { }_{0} G_{2}^{\mathbb{C}}\left[\begin{array}{c}
a_{1}\left|a_{1}^{\prime}, a_{2}\right| a_{2}^{\prime} ; z \\
-
\end{array}\right]=2 \Gamma^{\mathbb{C}}\left(a_{2}-a_{1} \mid a_{2}^{\prime}-a_{1}^{\prime}\right) z^{a_{1} \mid a_{1}^{\prime}}{ }_{0} F_{1}\left[\begin{array}{c}
- \\
1+a_{1}-a_{2}
\end{array} ; z\right]{ }_{0} F_{1}\left[\begin{array}{c}
- \\
1+a_{1}^{\prime}-a_{2}^{\prime} ; \bar{z}
\end{array}\right] \\
& +\left\{\text { similar term obtained by the transposition } a_{1}\left|a_{1}^{\prime} \longleftrightarrow a_{2}\right| a_{2}^{\prime}\right\} \text {. }
\end{aligned}
$$




\subsubsection{The Gauss hypergeometric functions}

For $p=q=2$

$$
\begin{aligned}
{ }_{2} G_{2}^{\mathbb{C}}\left[\begin{array}{c}
\left.a_{1}\left|a_{1}^{\prime}, a_{2}\right| a_{2}^{\prime} ; z\right]= \\
b_{1}\left|b_{1}^{\prime}, b_{1}\right| b_{1} ; z
\end{array}\right. & z^{a_{1} \mid a_{1}^{\prime}} \Gamma^{\mathbb{C}}\left(a_{2}-a_{1} \mid a_{2}^{\prime}-a_{1}^{\prime}\right) \prod_{\beta=1}^{2} \Gamma^{\mathbb{C}}\left(b_{\beta}+a_{1} \mid b_{\beta}^{\prime}+a_{1}^{\prime}\right) \\
& \times{ }_{2} F_{1}\left[\begin{array}{c}
b_{1}+a_{1}, b_{2}+a_{1} \\
1+a_{1}-a_{2}
\end{array} ; z\right]{ }_{2} F_{1}\left[\begin{array}{c}
b_{1}^{\prime}+a_{1}^{\prime}, b_{2}^{\prime}+a_{1}^{\prime} ; z \\
1+a_{1}^{\prime}-a_{2}^{\prime}
\end{array}\right] \\
& +\left\{\text { similar term obtained by the transposition } a_{1}\left|a_{1}^{\prime} \longleftrightarrow a_{2}\right| a_{2}^{\prime}\right\} .
\end{aligned}
$$

On the other hand Gelfand, Graev and Retakh [16] defined the analog of the Gauss hypergeometric function by the Euler integral (see the detailed examination in [33, Section 3]):

$$
\begin{aligned}
& { }_{2} F_{1}^{\mathbb{C}}\left[\begin{array}{c}
A\left|A^{\prime}, B\right| B^{\prime} \\
C \mid C^{\prime} ; z
\end{array} ;:=\frac{\Gamma^{\mathbb{C}}\left(B \mid B^{\prime}\right)}{\Gamma^{\mathbb{C}}\left(B \mid B^{\prime}\right) \Gamma^{\mathbb{C}}\left(C-B \mid C^{\prime}-B^{\prime}\right)}\right. \\
& \times \int_{\mathbb{C}} t^{B-1 \mid B^{\prime}-1}(1-t)^{C-B-1 \mid C^{\prime}-B^{\prime}-1}(1-z t)^{-A \mid-A^{\prime}} \mathrm{d} \overline{\bar{t}} .
\end{aligned}
$$

We have

$$
{ }_{2} F_{1}^{\mathbb{C}}\left[\begin{array}{c}
A\left|A^{\prime}, B\right| B^{\prime} \\
C \mid C^{\prime}
\end{array} ; z\right]=\frac{\Gamma\left(C \mid C^{\prime}\right)(-1)^{C-C^{\prime}}}{\Gamma\left(A \mid A^{\prime}\right) \Gamma\left(B \mid B^{\prime}\right)}{ }_{2} F_{2}\left[\begin{array}{c}
0|0,1-C| 1-C^{\prime} \\
A\left|A^{\prime}, B\right| B^{\prime}
\end{array} ; z\right] .
$$

We do not know which notation is better. In any case, for the notation ${ }_{2} F_{1}^{\mathbb{C}}$ formulas are precisely parallel to the classical theory of the Gauss hypergeometric functions.

As for the Kummer and Bessel cases the system (1.19) has 4-dimensional space of local solutions and a two-dimensional subspace of solutions that are non-ramified at 0 . The function ${ }_{2} F_{1}^{\mathbb{C}}$ is selected from this subspace by the condition of non-ramification at $z=1$, see [33, Proposition 3.11].

\subsection{Some simple properties of the functions ${ }_{p} G_{q}^{\mathbb{C}}$}

Here we imitate properties of the Meijer $G$-function (see Prudnikov, Brychkov and Marichev [40, Vol. 3, Section 8.2]). Clearly, the function ${ }_{p} G_{q}^{\mathbb{C}}$ is symmetric with respect to permutations of $a_{1}\left|a_{1}^{\prime}, \ldots, a_{p}\right| a_{p}^{\prime}$ and of $b_{1}\left|b_{1}^{\prime}, \ldots, b_{q}\right| b_{q}^{\prime}$. If $a_{m}-a_{l} \in \mathbb{Z}$, then we have an additional symmetry

$$
{ }_{p} G_{q}^{\mathbb{C}}\left[\begin{array}{c}
\left(a \mid a^{\prime}\right) \\
\left(b \mid b^{\prime}\right)
\end{array} ; z\right]={ }_{p} G_{q}^{\mathbb{C}}\left[\begin{array}{c}
\left(a \mid a^{\prime}\right) \backslash m, l \\
\left(b \mid b^{\prime}\right)
\end{array}\left|a_{l}^{\prime}, a_{m}^{\prime}\right| a_{l} ; z\right], \quad \text { if } a_{k}-a_{l} \in \mathbb{Z}
$$

(if $a_{k}-a_{l} \notin \mathbb{Z}$, then the right hand side makes no sense), this follows from (1.7).

Changing the variables $k \mapsto-k$ and $\sigma \mapsto-\sigma$ in (1.15) we get

$$
{ }_{p} G_{q}^{\mathbb{C}}\left[\begin{array}{c}
\left(a \mid a^{\prime}\right) \\
\left(b \mid b^{\prime}\right)
\end{array} ; z\right]={ }_{q} G_{p}^{\mathbb{C}}\left[\begin{array}{l}
\left(b \mid b^{\prime}\right) \\
\left(a \mid a^{\prime}\right)
\end{array} ; z^{-1}\right] .
$$

Changing only the summation index $k \mapsto-k$, we get

$$
{ }_{p} G_{q}^{\mathbb{C}}\left[\begin{array}{c}
\left(a \mid a^{\prime}\right) \\
\left(b \mid b^{\prime}\right)
\end{array} ; z\right]=(-1)^{\sum\left(a_{\alpha}-a_{\alpha}^{\prime}\right)+\sum\left(b_{\beta}-b_{\beta}^{\prime}\right)}{ }_{p} G_{q}^{\mathbb{C}}\left[\begin{array}{c}
\left(a \mid a^{\prime}\right) \\
\left(b \mid b^{\prime}\right)
\end{array} ; \bar{z}\right] .
$$

Keeping in the mind the reflection formula (1.8b), we get

$$
{ }_{p+1} G_{q+1}^{\mathbb{C}}\left[\begin{array}{l}
\left(a \mid a^{\prime}\right), \quad c \mid c^{\prime} \\
\left(b \mid b^{\prime}\right), 1-c \mid 1-c^{\prime}
\end{array} ; z\right]=(-1)^{c-c^{\prime}}{ }_{p} G_{q}^{\mathbb{C}}\left[\begin{array}{c}
\left(a \mid a^{\prime}\right) \\
\left(b \mid b^{\prime}\right)
\end{array} ; z\right] .
$$


Shifting variables $k \mapsto k+l, \sigma \mapsto \sigma+\tau$, we come to

$$
z^{c \mid c^{\prime}}{ }_{p} G_{q}^{\mathbb{C}}\left[\begin{array}{c}
\left(a \mid a^{\prime}\right) \\
\left(b \mid b^{\prime}\right)
\end{array} z\right]={ }_{p} G_{q}^{\mathbb{C}}\left[\begin{array}{c}
\left(a \mid a^{\prime}\right)+c \mid c^{\prime} \\
\left(b \mid b^{\prime}\right)-c \mid c^{\prime}
\end{array} ; z\right]
$$

Keeping in mind (1.8b), we obtain

$$
\begin{aligned}
& { }_{m p} G_{m q}^{\mathbb{C}}\left[\begin{array}{c}
\left(a \mid a^{\prime}\right),\left(a \mid a^{\prime}\right)+\frac{1}{m}\left|\frac{1}{m}, \ldots,\left(a \mid a^{\prime}\right)+\frac{m-1}{m}\right| \frac{m-1}{m} \\
\left(b \mid b^{\prime}\right),\left(b \mid b^{\prime}\right)+\frac{1}{m}\left|\frac{1}{m}, \ldots,\left(b \mid b^{\prime}\right)+\frac{m-1}{m}\right| \frac{m-1}{m} ; z
\end{array} ;\right. \\
& =m^{p+q-2-\sum\left(a_{\alpha}+a_{\alpha}^{\prime}\right)-\sum\left(b_{\beta}+b_{\beta}^{\prime}\right)} \sum_{l=0}^{m-1}{ }_{p} G_{q}^{\mathbb{C}}\left[\begin{array}{c}
\left(m a \mid m a^{\prime}\right) \\
\left(m b \mid m b^{\prime}\right)
\end{array} ; \mathrm{e}^{2 \pi \mathrm{i} l / m} z^{1 / m} m^{m p(-q)}\right],
\end{aligned}
$$

here we have a summation, which is absent for the classical Meijer $G$-function.

Differentiating the integral by the parameter $z$ we get

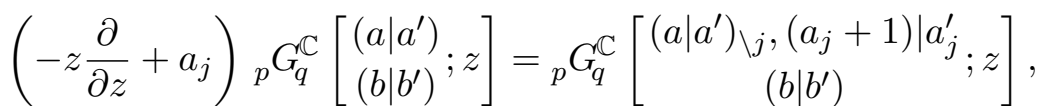

$$
\begin{aligned}
& \left(z \frac{\partial}{\partial z}+b_{m}\right){ }_{p} G_{q}^{\mathbb{C}}\left[\begin{array}{c}
\left(a \mid a^{\prime}\right) \\
\left(b \mid b^{\prime}\right)
\end{array} ; z\right]={ }_{p} G_{q}^{\mathbb{C}}\left[\begin{array}{c}
\left(a \mid a^{\prime}\right) \\
\left(b \mid b^{\prime}\right)_{\backslash m},\left(b_{m}+1\right) \mid b_{m}^{\prime}
\end{array} ; z\right] .
\end{aligned}
$$

Due to the $(-1)$ in (1.8c), the similar equations for $\bar{z} \frac{\partial}{\partial \bar{z}}$ differ from (3.10a) and (3.10b) by signs

$$
\begin{aligned}
& \left(-\bar{z} \frac{\partial}{\partial \bar{z}}+a_{j}^{\prime}\right){ }_{p} G_{q}^{\mathbb{C}}\left[\begin{array}{c}
\left(a \mid a^{\prime}\right) \\
\left(b \mid b^{\prime}\right)
\end{array} ; z\right]=-{ }_{p} G_{q}^{\mathbb{C}}\left[\begin{array}{c}
\left(a \mid a^{\prime}\right)_{\backslash j}, a_{j} \mid\left(a_{j}^{\prime}+1\right) \\
\left(b \mid b^{\prime}\right)
\end{array} ; z\right], \\
& \left(\bar{z} \frac{\partial}{\partial \bar{z}}+b_{m}^{\prime}\right){ }_{p} G_{q}^{\mathbb{C}}\left[\begin{array}{c}
\left(a \mid a^{\prime}\right) \\
\left(b \mid b^{\prime}\right)
\end{array} ; z\right]=-{ }_{p} G_{q}^{\mathbb{C}}\left[\begin{array}{c}
\left(a \mid a^{\prime}\right) \\
\left(b \mid b^{\prime}\right)_{\backslash m}, b_{m} \mid\left(b_{m}^{\prime}+1\right)
\end{array} ; z\right] .
\end{aligned}
$$

The last four equalities (3.10a)-(3.11b) imply the differential equations (1.19)-(1.20b).

Also

$$
\begin{aligned}
{ }_{p} G_{q}^{\mathbb{C}} & {\left[\begin{array}{c}
\left.\left(a \mid a^{\prime}\right)_{\backslash j},\left(a_{j}+1\right) \mid a_{j}^{\prime} ; z\right]-{ }_{p} G_{q}^{\mathbb{C}} \\
\left(b \mid b^{\prime}\right)
\end{array}\right.} \\
& \left.=\left(a_{j}-a_{m}\right)_{p} G_{q}^{\mathbb{C}}\left[\begin{array}{c}
\left(a \mid a^{\prime}\right) \backslash m,\left(a_{m}^{\prime}\right) \\
\left(b \mid b^{\prime}\right)
\end{array} ; z\right], \mid a_{m}^{\prime} ; z\right]
\end{aligned}
$$

and

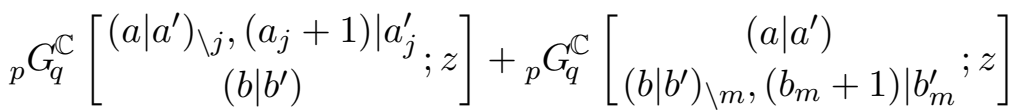

$$
\begin{aligned}
& =\left(a_{j}+b_{m}\right)_{p} G_{q}^{\mathbb{C}}\left[\begin{array}{l}
\left(a \mid a^{\prime}\right) \\
\left(b \mid b^{\prime}\right)
\end{array} ; z\right] .
\end{aligned}
$$

\subsection{References, links and problems}

(1). Gauss hypergeometric functions of the complex field (our ${ }_{2} G_{2}^{\mathbb{C}}$ modulo a simple factor) were defined by Gelfand, Graev and Retakh in [16] by the Euler integral (3.5). Many formulas for such functions were obtained in [33, Section 3].

(2). Marichev [31] proposed the following trick, which became the main tool in the creation of the Prudnikov, Brychkov and Marichev tables [40], ${ }^{6}$ see also comments in [41]. Many special functions (and many elementary functions) are special cases of the Meijer $G$-functions, i.e., can

\footnotetext{
${ }^{6}$ See also the tables [4].
} 
be written as Mellin-Barnes integrals (2.1). Therefore they are inverse Mellin transforms of products

$$
\frac{\prod_{\alpha=1}^{A} \Gamma\left(A_{\alpha}+\sigma\right) \prod_{\beta=1}^{B} \Gamma\left(B_{\beta}-\sigma\right)}{\prod_{\gamma=1}^{C} \Gamma\left(C_{\gamma}+\sigma\right) \prod_{\delta=1}^{D} \Gamma\left(D_{\delta}-\sigma\right)} .
$$

Take two such functions $\Phi(x), \Psi(x)$. Then we can evaluate the convolution

$$
\Theta(x)=\int_{0}^{\infty} \Phi(y) \Psi(x / y) y^{-1} \mathrm{~d} y .
$$

Indeed, the Mellin transform of $\Theta$ is the product of Mellin transforms, therefore we get a product of two functions of the type (3.12), i.e., a function of the same type. Now we can express $\Theta(x)$ as a linear combination of hypergeometric functions.

Numerous formulas in tables of integrals (such as Gradshteyn and Ryzhik [18]) whose initial derivations were ingenious can be obtained in this straightforward way. The table of evaluations of $G$-functions in Prudnikov, Brychkov and Marichev [40, Vol. 3, Section 8.4] contains 90 pages, for each pair of lines we can apply this trick. ${ }^{7}$

Our Theorem 1.7 with formulas (3.6), (3.7), (3.8) gives us the same tool. ${ }^{8}$ However, in our case the picture is less sophisticated. The classical Meijer functions depend on 4 subscripts and superscripts (see (2.1)), In our case the reflection formula (1.8b) allows to move $\Gamma^{\mathbb{C}}$-factors from the denominator to numerator. As a result, functions ${ }_{p} G_{q}^{\mathbb{C}}$ depend only on two subscripts $p, q$.

Apparently, most ${ }^{9}$ of identities for classical hypergeometric functions as they are exposed in $[13,14]$ (Chapters $2,4,6,7),[1,43]$ have counterparts for functions ${ }_{p} G_{q}^{\mathbb{C}}$, but different classical formulas can have the same counterpart (for instance the ${ }_{5} H_{5}$-Dougall formula and the de Branges-Wilson integral correspond to one integral over $\mathbb{Z} \times \mathbb{R}$, see [39]).

(3). A collection of beta-integrals involving products of $\Gamma^{\mathbb{C}}$ is known, see Bazhanov, Mangazeev and Segeev [2], Kels [27, 28], Derkachov, Manashov and Valinevich [6, 8, 9, 10], Neretin [39], Sarkissian and Spiridonov [42], in particular, this collection contains counterparts of the de Branges-Wilson integral and the Nassrallah-Rahman integral. Such integrals can be regarded as evaluations of functions ${ }_{p} G_{p}^{\mathbb{C}}(z)$ at the point $(-1)^{p}$.

(4). Such beta integrals and such hypergeometric functions arise as limits of elliptic betaintegrals and the hypergeometric functions ${ }_{p} G_{p}^{\mathbb{C}}$ as limits of elliptic hypergeometric functions, see Sarkissian and Spiridonov [42].

(5). The classical expansion in Jacobi polynomials has a well-known continuous analog known under terms 'Olevski transform', 'generalized Mehler-Fock transform', 'Jacobi transform', see, e.g., [29]; there is also a second continuous analog [38]. The paper Molchanov and Neretin [33] contains a complex counterpart of these 3 transformations (expansions in the Jacobi polynomials and two integral operators), it is a unitary integral transform with ${ }_{2} G_{2}^{\mathbb{C}}$-kernel acting from a certain weighted $L^{2}$ on $\mathbb{C}$ to a certain weighted $L^{2}$ on $\Lambda \simeq \mathbb{Z} \times \mathbb{R}$.

The 'Jacobi transform' is a representative of a big zoo of hypergeometric integral transforms (see, e.g., [46]), it is natural to think that their counterparts exist in ${ }_{p} G_{q}^{\mathbb{C}}$-cases. Integral transforms also can be applied as a tool for the production of special-functional identities (clearly, several transforms were used for the creation of [40, Vol. 3], on possibilities of the Jacobi transform, see [37]). An example of application of the ${ }_{2} G_{2}^{\mathbb{C}}$-transform is contained in [39].

\footnotetext{
${ }^{7}$ Chapter 7 of the same book (160 pages) also provides us a material for this business.

${ }^{8}$ Our arguments are not sufficient for integrals (1.22) with functions ${ }_{1} G_{0}^{\mathbb{C}}$, see (3.1). Apparently, the formula (1.22) remains valid in this case.

${ }^{9}$ With some exceptions, for instance an application of formula (3.9) can be problematic.
} 
(6). It is well-known that representation theory of the group $\operatorname{SL}(2, \mathbb{R})$ is closely related to theory of hypergeometric functions (as the Bessel functions, the confluent hypergeometric functions, the Gauss hypergeometric functions, ${ }_{3} F_{2}(1)$, and the balanced ${ }_{4} F_{3}(1)$ ). Application of harmonic analysis related to the Lorentz group ${ }^{10} \mathrm{SL}(2, \mathbb{C})$ to special functions are far not so popular (at least among pure mathematicians). However, if to ask such a question, then hypergeometric functions of complex field come thick and fast.

- a tensor product $\rho_{1} \otimes \rho_{2}$ of two irreducible unitary representations of $\operatorname{SL}(2, \mathbb{C})$ is a multiplicity free direct integral (see Naimark $[34,35,36]$ ). Therefore we can canonically decompose a triple tensor product

$$
\left(\rho_{1} \otimes \rho_{2}\right) \otimes \rho_{3}=\rho_{1} \otimes\left(\rho_{2} \otimes \rho_{3}\right)
$$

in two ways. In the first case we decompose $\rho_{1} \otimes \rho_{2}$ and multiply each component by $\rho_{3}$, in the second case we start from $\rho_{2} \otimes \rho_{3}$. Realizing this idea ${ }^{11}$ we get two explicit decompositions of the same representation, the intertwining operator splits into a direct integral of intertwining operators acting in isotypic components, such operators can be regarded as counterparts of Racah coefficients (6j-symbols). Ismagilov [23, 24] (see also Derkachov iand Spiridonov [11]) showed that such 'Racah operators' are integrals transforms whose kernels have a form ${ }_{4} G_{4}^{\mathbb{C}}(1)$. The 'Racah operators' are $G^{\mathbb{C}}$-counterparts of expansions in Racah polynomials, expansions in Wilson polynomials, and the 'Wilson function transforms' defined by Groenevelt [19, 20].

- Recall that the Lorentz group $\mathrm{SL}(2, \mathbb{C})$ is locally isomorphic to the complex orthogonal group $\mathrm{SO}(3, \mathbb{C})$. Consider the symmetric space $\mathrm{SO}(3, \mathbb{C}) / \mathrm{SO}(2, \mathbb{C})$, it can be regarded as a quadric $x^{2}+y^{2}+z^{2}=1$ in $\mathbb{C}^{3}$, or the complexification of the sphere $x^{2}+y^{2}+z^{2}=1$ in $\mathbb{R}^{3}$. Under the complexification, the Laplacian on the real sphere splits into two commuting Laplacians, one is holomorphic, another is anti-holomorphic. A question about their joint spectral decomposition in a space of $\mathrm{SO}(2)$-invariant functions leads to ${ }_{2} G_{2}$-transform considered in Molchanov and Neretin [33].

The shortest way of appearance of ${ }_{2} G_{2}^{\mathbb{C}}$-functions is discussed in Section 3.4 below.

(7). Dotsenko and Fateev [12] obtained a complex version of the Selberg integral; Derkachov, Manashov and Valinevich $[9,10]$ obtained multi-dimensional beta-integrals with products of $\Gamma^{\mathbb{C}}$ functions (counterparts of the Gustafson's extension of the second Barnes lemma).

There arises a question about multi-dimensional symmetric $\mathbb{C}$-counterparts of the HeckmanOpdam hypergeometric functions [21]. The obvious candidates are spherical distributions on symmetric spaces $G_{\mathbb{C}} / K_{\mathbb{C}}$, where $G_{\mathbb{C}}$ is a complex semisimple Lie group and $K_{\mathbb{C}}$ a complex symmetric subgroup.

On the other hand there are no reasons to hope that in the multi-dimensional case complex spherical transforms are unitary operators (unexpectedly, the radial parts of Laplace operators can be non-commuting in the Nelson sense, see [33, Theorem 1.1]).

\subsection{The Vilenkin model for $\operatorname{SL}(2, \mathbb{C})$}

First, we modify notation. Let us denote elements of $\Lambda_{\mathbb{C}}$ by bold letters, denote $a \mid a^{\prime}$ by $\mathbf{a}, 1 \mid 1$ by $\mathbf{1}$, and $(-1)^{a-a^{\prime}}$ by $(-1)^{\mathbf{a}}$. Since $\Lambda \simeq \mathbb{Z} \times \mathbb{R}$, we can denote

$$
\int_{\Lambda}(\ldots)=\sum_{k \in \mathbb{Z}} \int_{\mathbb{R}}(\ldots) .
$$

\footnotetext{
${ }^{10}$ See old works Gelfand, Graev and Vilenkin [17], Naimark [34, 35, 36], Gelfand and Graev [15] on SL(2, C)harmonic analysis, see also Derkachov, Korchemsky and Manashov [5, 7].

${ }^{11}$ It is difficult to extend this approach to unitary representations of other groups since decompositions of tensor products usually have multiplicities $>1$ (even for $\mathrm{SL}(2, \mathbb{R})$ ). Several multiplicity free cases were examined in $[20,23,24,25,26]$.
} 
Now let us explain how the hypergeometric functions ${ }_{2} F_{1}^{\mathbb{C}}$ arise from representations of the Lorentz group $\mathrm{SL}(2, \mathbb{C})$. Recall that this group can be realized as the group of all complex $2 \times 2$ matrices $\left(\begin{array}{ll}a & b \\ c & d\end{array}\right)$ with determinant $a d-b c=1$. The principal series of representations $T_{\boldsymbol{\sigma}}$ of this group is parametrized by $\boldsymbol{\sigma}=\sigma \mid \sigma^{\prime} \in \Lambda_{\mathbb{C}}$. They act in the space of functions on $\mathbb{C}$ by operators

$$
T_{\boldsymbol{\sigma}}\left(\begin{array}{ll}
a & b \\
c & d
\end{array}\right) f(z):=f\left(\frac{b+z d}{a+z c}\right)(a+z c)^{-\mathbf{1}+\boldsymbol{\sigma}} .
$$

For $\sigma \in \Lambda$ we get unitary representations in $L^{2}(\mathbb{C})$, for details, see [17, Chapter III].

Let us realize the representations $T_{\boldsymbol{\sigma}}$ in a space of functions on $\Lambda$ conjugating them by the Mellin transform ${ }^{12}$

$$
\widetilde{T}_{\boldsymbol{\sigma}}\left(\begin{array}{ll}
a & b \\
c & d
\end{array}\right)=\mathcal{M} \circ T_{\boldsymbol{\sigma}}\left(\begin{array}{ll}
a & b \\
c & d
\end{array}\right) \circ \mathcal{M}^{-1} .
$$

A straightforward calculation shows that

$$
\widetilde{T}_{\boldsymbol{\sigma}}\left(\begin{array}{ll}
a & b \\
c & d
\end{array}\right) F(\mu)=\frac{1}{4 \pi^{2} \mathrm{i}} \int_{\Lambda} \mathcal{L}\left[\boldsymbol{\mu}, \boldsymbol{\lambda} ;\left(\begin{array}{ll}
a & b \\
c & d
\end{array}\right)\right] F(\boldsymbol{\lambda}) \mathrm{d} \boldsymbol{\lambda},
$$

where

$$
\mathcal{L}\left[\boldsymbol{\mu}, \boldsymbol{\lambda} ;\left(\begin{array}{ll}
a & b \\
c & d
\end{array}\right)\right]=\int_{\mathbb{C}} z^{\boldsymbol{\mu}-1}(a+z c)^{\boldsymbol{\sigma}-\boldsymbol{\lambda}-1}(b+z d)^{\boldsymbol{\lambda}} \mathrm{d} \overline{\bar{z}} .
$$

Substituting $z=-\frac{a}{c} u$ we come to

$$
\mathcal{L}\left[\boldsymbol{\mu}, \boldsymbol{\lambda} ;\left(\begin{array}{ll}
a & b \\
c & d
\end{array}\right)\right]=(-1)^{\boldsymbol{\mu}} a^{\boldsymbol{\sigma}+\boldsymbol{\mu}-\boldsymbol{\lambda}-\mathbf{1}} b^{\boldsymbol{\lambda}} c^{-\boldsymbol{\mu}} \frac{\Gamma^{\mathbb{C}}(\boldsymbol{\sigma}-\boldsymbol{\lambda}+\boldsymbol{\mu})}{\Gamma^{\mathbb{C}}(\boldsymbol{\mu}) \Gamma^{\mathbb{C}}(\boldsymbol{\sigma}-\boldsymbol{\lambda})}{ }_{2} F_{1}\left[\begin{array}{c}
\boldsymbol{\mu},-\boldsymbol{\lambda} \\
\boldsymbol{\sigma}-\boldsymbol{\lambda}+\boldsymbol{\mu} ; \frac{a d}{b c}
\end{array}\right]
$$

\section{Acknowledgements}

The work was supported by the grant FWF, P31591. I am grateful to M.I. Graev, V.F. Molchanov, V.A. Spiridonov, and S.É. Derkachov for discussions and references, I also thank the referees for useful comments.

\section{References}

[1] Andrews G.E., Askey R., Roy R., Special functions, Encyclopedia of Mathematics and its Applications, Vol. 71, Cambridge University Press, Cambridge, 1999.

[2] Bazhanov V.V., Mangazeev V.V., Sergeev S.M., Exact solution of the Faddeev-Volkov model, Phys. Lett. A 372 (2008), 1547-1550, arXiv:0706.3077.

[3] Beals R., Wong R., Special functions and orthogonal polynomials, Cambridge Studies in Advanced Mathematics, Vol. 153, Cambridge University Press, Cambridge, 2016.

[4] Brychkov Yu.A., Marichev O.I., Savischenko N.V., Handbook of Mellin transforms, Advances in Applied Mathematics, CRC Press, Boca Raton, FL, 2019.

[5] Derkachov S.É., Korchemsky G.P., Manashov A.N., Noncompact Heisenberg spin magnets from high-energy QCD. I. Baxter Q-operator and separation of variables, Nuclear Phys. B 617 (2001), 375-440, arXiv:hepth/0107193.

[6] Derkachov S.É., Manashov A.N., $\mathcal{R}$-matrix and Baxter $\mathcal{Q}$-operators for the noncompact $\operatorname{SL}(N, \mathbb{C})$ invariant spin chain, SIGMA 2 (2006), 084, 20 pages, arXiv:nlin.SI/0612003.

[7] Derkachov S.É., Manashov A.N., General solution of the Yang-Baxter equation with the symmetry group $\mathrm{SL}(n, \mathbb{C})$, St. Petersburg Math. J. 21 (2010), 513-577.

\footnotetext{
${ }^{12}$ Cf. a model of representations of $\operatorname{SL}(2, \mathbb{R})$ in Vilenkin [45, Section VII.3].
} 
[8] Derkachov S.E., Manashov A.N., On complex gamma-function integrals, SIGMA 16 (2020), 003, 20 pages, arXiv:1908.01530.

[9] Derkachov S.É., Manashov A.N., Valinevich P.A., Gustafson integrals for $\operatorname{SL}(2, \mathbb{C})$ spin magnet, J. Phys. A: Math. Theor. 50 (2017), 294007, 12 pages, arXiv:1612.00727.

[10] Derkachov S.É., Manashov A.N., Valinevich P.A., SL(2, C) Gustafson integrals, SIGMA 14 (2018), 030, 16 pages, arXiv:1711.07822.

[11] Derkachov S.É., Spiridonov V.P., The $6 j$-symbols for the $\mathrm{SL}(2, \mathbb{C})$ group, Theoret. and Math. Phys. 198 (2019), 29-47, arXiv:1711.07073.

[12] Dotsenko V.S., Fateev V.A., Four-point correlation functions and the operator algebra in 2D conformal invariant theories with central charge $C \leq 1$, Nuclear Phys. B 251 (1985), 691-734.

[13] Erdélyi A., Magnus W., Oberhettinger F., Tricomi F.G., Higher transcendental functions, Vol. I, McGrawHill Book Company, Inc., New York - Toronto - London, 1953.

[14] Erdélyi A., Magnus W., Oberhettinger F., Tricomi F.G., Higher transcendental functions, Vol. II, McGrawHill Book Company, Inc., New York - Toronto - London, 1953.

[15] Gel'fand I.M., Graev M.I., An application of the horisphere method to the spectral analysis of functions in real and imaginary Lobatchevsky spaces, Tr. Mosk. Mat. Obs. 11 (1962), 243-308.

[16] Gel'fand I.M., Graev M.I., Retakh V.S., Hypergeometric functions over an arbitrary field, Russian Math. Surveys 59 (2004), 831-905.

[17] Gel'fand I.M., Graev M.I., Vilenkin N.Y., Generalized functions, Vol. 5, Integral geometry and representation theory, Academic Press, New York - London, 1966.

[18] Gradshteyn I.S., Ryzhik I.M., Table of integrals, series, and products, 4th ed., Academic Press, New York London, 1965.

[19] Groenevelt W., The Wilson function transform, Int. Math. Res. Not. 2003 (2003), 2779-2817, arXiv:math.CA/0306424.

[20] Groenevelt W., Wilson function transforms related to Racah coefficients, Acta Appl. Math. 91 (2006), 133191, arXiv:math.CA/0501511.

[21] Heckman G.J., Opdam E.M., Root systems and hypergeometric functions. I, Compositio Math. 64 (1987), 329-352.

[22] Hörmander L., The analysis of linear partial differential operators. I. Distribution theory and Fourier analysis, Grundlehren der Mathematischen Wissenschaften, Vol. 256, Springer-Verlag, Berlin, 1983.

[23] Ismagilov R.S., On Racah operators, Funct. Anal. Appl. 40 (2006), 222-224.

[24] Ismagilov R.S., Racah operators for principal series of representations of the group SL(2, C), Sb. Math. 198 (2007), 369-381.

[25] Ismagilov R.S., Racah operators for the group of motions, Funct. Anal. Appl. 42 (2008), 72-74.

[26] Ismagilov R.S., Racah operators for unitary representations of groups, Mosc. Math. J. 14 (2014), 565-576.

[27] Kels A.P., New solutions of the star-triangle relation, J. Phys. A: Math. Theor. 47 (2014), 055203, 7 pages, arXiv:1302.3025.

[28] Kels A.P., New solutions of the star-triangle relation with discrete and continuous spin variables, J. Phys. A: Math. Theor. 48 (2015), 435201, 19 pages, arXiv:1504.07074.

[29] Koornwinder T.H., Jacobi functions and analysis on noncompact semisimple Lie groups, in Special Functions: Group Theoretical Aspects and Applications, Editors Askey R.A., Koornwinder T.H., Schempp W.J., Math. Appl., Reidel, Dordrecht, 1984, 1-85.

[30] Luke Y.L., The special functions and their approximations, Vol. I, Mathematics in Science and Engineering, Vol. 53, Academic Press, New York - London, 1969.

[31] Marichev O.I., Handbook of integral transforms of higher transcendental functions. Theory and algorithmic tables, Ellis Horwood Series: Mathematics and its Applications, Ellis Horwood Ltd., Chichester, 1983 (translated from: A method of calculating integrals from special functions (theory and tables of formulas), Nauka i Tekhnika, Minsk, 1978).

[32] Mimachi K., Complex hypergeometric integrals, in Representation Theory, Special Functions and Painlevé equations - RIMS 2015, Adv. Stud. Pure Math., Vol. 76, Editors H. Konno, H. Sakai, J. Shiraishi, T. Suzuki, Y. Yamada, Math. Soc. Japan, Tokyo, 2018, 469-485. 
[33] Molchanov V.F., Neretin Yu.A., A pair of commuting hypergeometric operators on the complex plane and bispectrality, J. Spectr. Theory, to appear, arXiv:1812.06766.

[34] Naimark M.A., Decomposition of a tensor product of irreducible representations of the proper Lorentz group into irreducible representations. I. The case of a tensor product of representations of the fundamental series, Amer. Math. Soc. Transl. Ser. 2, Vol. 36, Amer. Math. Soc., Providence, RI, 1964, 101-136 (translated from: Tr. Mosk. Mat. Obs. 8 (1959), 121-153).

[35] Naimark M.A., Decomposition of a tensor product of irreducible representations of the proper Lorentz group into irreducible representations. II. The case of a tensor product of representations of the fundamental and complementary series, Amer. Math. Soc. Transl. Ser. 2, Vol. 36, Amer. Math. Soc., Providence, RI, 1964, 137-187 (translated from: Tr. Mosk. Mat. Obs. 9 (1960), 237-282).

[36] Naimark M.A., Decomposition of a tensor product of irreducible representations of the proper Lorentz group into irreducible representations. III. The case of a tensor product of representations of the complementary series, Amer. Math. Soc. Transl. Ser. 2, Vol. 36, Amer. Math. Soc., Providence, RI, 1964, 189-229 (translated from: Tr. Mosk. Mat. Obs. 10 (1961), 181-216).

[37] Neretin Yu.A., Beta-integrals and finite orthogonal systems of Wilson polynomials, Sb. Math. 193 (2002), 1071-1089, arXiv:math.CA/0206199.

[38] Neretin Yu.A., Some continuous analogues of the expansion in Jacobi polynomials, and vector-valued orthogonal bases, Funct. Anal. Appl. 39 (2005), 106-119, arXiv:math.CA/0309445.

[39] Neretin Yu.A., An analog of the Dougall formula and of the de Branges-Wilson integral, Ramanujan J., to appear, arXiv:1812.07341.

[40] Prudnikov A.P., Brychkov Yu.A., Marichev O.I., Integrals and series. Vols. 1-5. Gordon and Breach Science Publishers, New York, 1986-1992.

[41] Prudnikov A.P., Brychkov Yu.A., Marichev O.I., Calculation of integrals and the Mellin transform, J. Soviet Math. 54 (1991), 1239-1341 (translated from: Itogi Nauki i Tekhn. Ser. Mat. Anal., 27 (1989), 3-146).

[42] Sarkissian G.A., Spiridonov V.P., The endless beta integrals, SIGMA 16 (2020), 074, 21 pages, arXiv:2005.01059

[43] Slater L.J., Generalized hypergeometric functions, Cambridge University Press, Cambridge, 1966.

[44] Titchmarsh E.C., The theory of functions, 2nd ed., Oxford University Press, Oxford, 1939.

[45] Vilenkin N.Ja., Special functions and the theory of group representations, Translations of Mathematical Monographs, Vol. 22, Amer. Math. Soc., Providence, RI, 1968.

[46] Yakubovich S.B., Index transforms, World Sci. Publ. Co., Inc., River Edge, NJ, 1996. 